\title{
Oral Administration of Cannabis and $\Delta$-9-tetrahydrocannabinol (THC) Preparations: A Systematic Review
}

\author{
Lourdes Poyatos 1,2,+(D), Ana Pilar Pérez-Acevedo 1,2,*,+, Esther Papaseit 1,2, ${ }^{\dagger}$, \\ Clara Pérez-Mañá ${ }^{1,2,+}$, Soraya Martin ${ }^{1}$, Olga Hladun ${ }^{1,2}$, Adrià Siles ${ }^{3}$, Marta Torrens ${ }^{2,4}$, \\ Francesco Paolo Busardo ${ }^{5, \ddagger}$ and Magí Farré $1,2, \ddagger$ (D) \\ 1 Clinical Pharmacology Department, Hospital Universitari Germans Trias i Pujol and Institut de Recerca \\ Germans Trias (HUGTiP-IGTP), 08916 Badalona, Spain; lpoyatos@igtp.cat (L.P.); \\ epapaseit.germanstrias@gencat.cat (E.P.); cperezm.mn.ics@gencat.cat (C.P.-M.); \\ smartins.mn.ics@gencat.cat (S.M.); ohladun.germanstrias@gencat.cat (O.H.); \\ mfarre.germanstrias@gencat.cat (M.F.) \\ 2 Departments of Pharmacology, Therapeutics and Toxicology and Department of Psychiatry, \\ Universitat Autònoma de Barcelona, 08193 Cerdanyola del Vallés, Spain; mtorrens@parcdesalutmar.cat \\ 3 Pharmacy Department, Hospital Universitari Germans Trias i Pujol and Institut de Recerca Germans \\ Trias (HUGTiP-IGTP), 08916 Badalona, Spain; asiles@igtp.cat \\ 4 Drug Addiction Program, Institut de Neuropsiquiatria, Parc de Salut Mar and Institut Hospital del Mar de \\ Recerca Mèdica (PSMAR-IMIM), 08003 Barcelona, Spain \\ 5 Department of Excellence-Biomedical Sciences and Public Health, Università Politecnica delle Marche, \\ 60121 Ancona, Italy; f.p.busardo@staff.univpm.it \\ * Correspondence: appereza.germanstrias@gencat.cat; Tel.: +34-934978865 \\ + These authors shared first authorship. \\ $\ddagger$ These authors shared senior authorship.
}

Received: 15 May 2020; Accepted: 17 June 2020; Published: 23 June 2020

Abstract: Background and objective: Changes in cannabis legalization regimes in several countries have influenced the diversification of cannabis use. There is an ever-increasing number of cannabis forms available, which are gaining popularity for both recreational and therapeutic use. From a therapeutic perspective, oral cannabis containing $\Delta$-9-tetrahydrocannabinol (THC) and cannabidiol (CBD) is a promising route of administration but there is still little information about its pharmacokinetics (PK) effects in humans. The purpose of this systematic review is to provide a general overview of the available PK data on cannabis and THC after oral administration. Materials and Methods: A search of the published literature was conducted using the PubMed database to collect available articles describing the PK data of THC after oral administration in humans. Results: The literature search yielded 363 results, 26 of which met our inclusion criteria. The PK of oral THC has been studied using capsules (including oil content), tablets, baked goods (brownies and cookies), and oil and tea (decoctions). Capsules and tablets, which mainly correspond to pharmaceutical forms, were found to be the oral formulations most commonly studied. Overall, the results reflect the high variability in the THC absorption of oral formulations, with delayed peak plasma concentrations compared to other routes of administration. Conclusions: Oral THC has a highly variable PK profile that differs between formulations, with seemingly higher variability in baked goods and oil forms. Overall, there is limited information available in this field. Therefore, further investigations are required to unravel the unpredictability of oral THC administration to increase the effectiveness and safety of oral formulations in medicinal use.

Keywords: oral cannabis; oral THC; medical use; cannabis edibles; pharmacokinetics 


\section{Introduction}

\subsection{Cannabinoids}

Cannabis is the most widely used illicit drug worldwide, only surpassed by alcohol and tobacco when also considering legal substances. Recent investigations have highlighted the therapeutic potential of cannabis, resulting in a resurgence of its consumption for medical purposes. Although cannabis continues to be used mostly for recreational purposes, people increasingly consume it to benefit from its therapeutic properties [1-3].

$\Delta$-9-Tetrahydrocannabinol (THC) is the principal source of the psychoactive effects associated with cannabis use [3]. These effects result from the activity of THC as a partial agonist of the cannabinoid receptor CB1, which is primarily located in the central nervous system, and CB2, which is predominantly expressed in the peripheral tissues [4]. THC has observable effects on behavior, nociception, and appetite, as well as anti-inflammatory, antitumor, and antiemetic properties. THC is also responsible for the psychotropic effects and addictive and reinforcing properties of cannabis [5].

The other predominant component in the cannabis plant is cannabidiol (CBD), which is the primary cannabinoid in fiber-type hemps. Unlike THC, CBD does not have a direct effect on the receptors (CB1 and CB2) responsible for cannabis' psychoactive effects [6-8], although recent evidence has demonstrated the negative allosteric activity of CBD on CB1 [9].

CBD is believed to attenuate THC's psychotropic effects, thereby enhancing the safety (or safety profile) of cannabis products containing both cannabinoids. However, this interaction is not fully understood. Previous studies found that CBD does not alter THC's subjective effects [10-12], but a recent study reported an increase in plasma THC concentration and a slight exacerbation of THC-induced impairment in the presence of CBD [13].

Other cannabinoids may play a role in the overall effects of cannabis, such as $\Delta$-8-tetrahydrocannabinol, cannabinol (CBN), cannabicyclol (CBL), cannabichromene (CBC), and cannabigerol (CBG). However, these cannabinoids have fewer psychotropic effects than THC [14].

\subsection{Therapeutic Uses of Cannabis}

Cannabinoids exert most of their biological effects through interactions with the endocannabinoid system. Their wide range of effects makes cannabinoids good candidates for treating many ailments, including nausea, loss of appetite, neuropathic pain spasticity, epilepsy, chronic pain, etc. [5]. However, cannabinoids are currently typically prescribed as adjuvant treatments or after a patient does not respond well to first-line treatments.

The number of countries that have legalized therapeutic cannabis use (medical cannabis) has grown in recent years. In the Netherlands, the drastic increase in the prevalence of medical cannabis prescriptions can be explained by the emergence of new formulations, especially cannabis oils [15], which have become the preferred option for therapeutic use [16].

When considering the purpose of consumption, for medical cannabis users, edible cannabis is one of the most common consumption modes, along with vaporization [17-19], contrary to recreational users, who are more likely to smoke or vaporize [18].

\subsection{Oral Cannabis and THC and Other Routes of Administration}

Recreational cannabis is mainly consumed by smoking, which involves combusting the herbal cannabis present in a joint, blunt, pipe, bubbler, or bong/water pipe, among other forms. The psychoactive effects of THC appear in less than a minute after consumption. From the limited evidence available, it appears that smoking cannabis may be associated with respiratory diseases, as smoked cannabis contains several toxins and carcinogens also found in tobacco smoke [20]. No country that has authorized the medical use of cannabis recommends smoking as a method of consumption.

An alternative inhalation-based form that has become popular in recent years is using a vaporizer. This technique is considered less noxious than regular smoking, as vaporizing does not produce 
the pyrolytic compounds derived from combustion of the dried herb or extract, such as polycyclic aromatic hydrocarbons. However, vaporizers have been recently associated with acute respiratory illness, now referred to as e-cigarette or vaping product-use associated lung injury (EVALI) [21,22]. The cause of this condition is currently under investigation, although there is evidence implicating the vitamin $\mathrm{E}$ acetate used as a diluent in vaporizer liquids [23].

Changes in cannabis legalization in several countries have influenced the emergence of a variety of edible products containing cannabis, which have increasing popularity. At present, edible products are now available in new formats resembling sugary snacks (hard and soft candies) and baked goods (brownies, cookies), which appeal especially to young people. From a therapeutic perspective, oral cannabis intake is promising due to its long-lasting drug effects, easy administration, and reduced toxicity derived from pyrolytic by-products. To date, the limited information available describes a slow and erratic absorption, seemingly showing higher bioavailability in oil formulations [24].

\subsection{Oral Cannabinoid Preparations}

Medical cannabis refers to a broad range of products and preparations that contain cannabis and cannabinoids for therapeutic purposes. Several medical products with marketing authorization contain THC as their main component, including dronabinol (synthetic THC), commercialized as oral capsules (Marinol ${ }^{\circledR}$ ) or as an oral solution (Syndros ${ }^{\circledR}$ ), and nabilone (a synthetic THC analogue), which is marketed as oral capsules (Cesamet ${ }^{\circledR}$ or Canemes ${ }^{\circledR}$ ). Nabiximols (Sativex ${ }^{\circledR}$ ), available as a buccal spray, also includes CBD in its formulation [14,24-26]. However, few cannabis products have sufficient evidence to obtain approval for therapeutic use in the USA and several European countries.

Formulations derived from the Cannabis sativa plant that do not have marketing authorization for medical use are known as "cannabis preparations". This term encompasses raw cannabis, compound preparations, and standardized cannabis preparations, which include cannabis flowers, granulates, and oil extracts [27].

In the Netherlands, cannabis is produced in five standardized strains that are commercially available and classified by their THC and CBD content [28]. In Italy, the Ministry of Health authorized the commercialization of standardized cannabis (FM2, 5-8\% THC and 7-12\% CBD) for medical purposes, which is produced by the Military Pharmaceutical Institute in Florence [29]. As per recommendations, this medical cannabis is consumed in decoctions or oils following standardized indications [30]. In 2018, a new strain of medical cannabis began production (FM1, 13-20\% THC and $<1 \%$ CBD), but no therapeutic indications are currently authorized for this new strain [31,32]. In Canada, medical cannabis is manufactured under a public license, mostly in the form of oil. Cannimed ${ }^{\circledR}$ can be purchased as oil capsules, oil, dried flowers, or in a topical form, allowing oral, vaporized, or topical administration $[33,34]$.

Edible cannabis is associated with a high rate of emergency department visits, mainly due to gastrointestinal symptoms, intoxication, and psychiatric effects [35]. The high variability of oral THC absorption and the delayed onset of effects can lead to the overconsumption of edible preparations, especially among naive users.

Considering that cannabinoids are proposed to alleviate a wide range of ailments, the identification and interpretation of their pharmacokinetics (PK) are essential for their use as pharmaceutical products. The purpose of this review was to examine the available data on THC PK after the oral administration of cannabis and THC, as reported in humans.

\section{Materials and Methods}

This systematic review was performed according to PRISMA (Preferred Reporting Items for Systematic Reviews and Meta-Analyses) guidelines [36].

Potential studies were systematically searched and identified by one of the authors (L.P.). The study selection was jointly conducted by two of the authors (L.P. and M.F.). After reading the summary of 
each study, in case of a disagreement, the final decision was reached by consensus. The data were collected by the author L.P. and reviewed by the other authors (M.F. and A.P.P.).

A search of the published literature was conducted using the PubMed database up to March 2020. The keywords used for the search were "oral cannabis", "edible cannabis", "oral THC", "pharmacokinetic", "blood collection", "dronabinol", "synthetic THC", "plasma levels", and "Cmax". To be included, studies had to follow a pharmacokinetic model, include at least the maximum plasma concentration values (Cmax) and time needed to reach the maximum concentrations (Tmax) as PK parameters, and examine oral administration, although they could also include other routes of administration. Single-dose studies were preferred, but multiple-dose studies were included if the blood was collected at various time points following initial dosing. Only articles whose abstracts met our selected criteria were selected. Animal studies, articles focusing on routes of administration other than oral, nabilone (a synthetic cannabinoid), nabiximols, and studies of CBD administration were excluded from the review. Studies featuring the administration of nabiximols were only included if their preparations were compared to those of other oral cannabis/THC preparations.

Only articles written in English were selected. All articles were reviewed independently by the authors to determine their relevance in the framework of the current study.

The following data were collected from the reviewed articles: Design of the study, number of participants, gender, previous experience with cannabis, route of administration, product containing THC, dose of THC, basic PK measurements (maximum concentration, or Cmax, and time to achieve maximum concentrations, or Tmax) in plasma or blood, and pharmacological effects (if measured).

In order to evaluate a relationship between the administered dose and peak concentrations (Cmax), Spearman correlations were conducted between doses of THC and Cmax values in each formulation group. A linear regression procedure that allows for the calculation of correlation coefficients was used. Analyses were performed using GraphPad Prism 5.

\section{Results}

The literature search yielded a total of 363 results, 26 of which met our inclusion criteria (Figure 1) [37-62]. These studies are summarized in Tables 1-4. Besides the oral administration route, cannabis was taken in by other routes in eight of the studies, including sublingual, respiratory/inhaled, buccal, intravenous, and oropharyngeal routes. THC can be present in various dosage forms, each with different PK properties, which are crucial to know for a molecule intended for therapeutic applications. The oral absorption of THC was studied using oil capsules, tablets, baked goods (brownies and cookies), oils, and decoctions. However, there was no information on other products containing cannabis, including candies and chocolates.

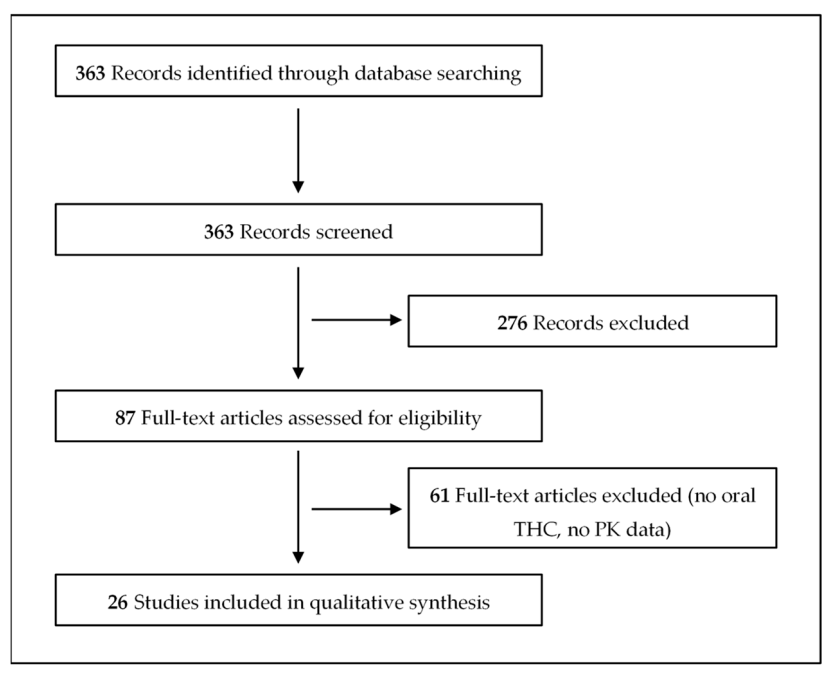

Figure 1. Flow chart for the study retrieval and selection. 
Table 1. Summary of studies included in this systematic review reporting the pharmacokinetic parameters of $\Delta$-9-tetrahydrocannabinol (THC) following capsule administration.

\begin{tabular}{|c|c|c|c|c|c|c|c|c|}
\hline References & Study Design & Participants & $\begin{array}{c}\text { Route of } \\
\text { Administration }\end{array}$ & Formulation & Dose & $\begin{array}{c}\text { Cmax (ng/mL) } \\
\text { Mean } \pm \text { SD, Median (Range) }\end{array}$ & $\operatorname{Tmax}(\mathrm{h})$ & Pharmacological Effects \\
\hline \multirow{4}{*}{ Wall et al., 1983 [37] } & \multirow{4}{*}{$\mathrm{OL}, \mathrm{NP}$} & \multirow{4}{*}{$6 \mathrm{M}, 6 \mathrm{~F}$} & \multirow[b]{2}{*}{ Oral } & \multirow[b]{2}{*}{ Capsules } & 20 mg THC (men) & $\begin{array}{c}\text { Men } \\
\text { THC: } 14 \pm 9.7^{\mathrm{b}} \\
11-\mathrm{OH}-\mathrm{THC}: 6.6 \pm 3.4^{\mathrm{b}}\end{array}$ & $\begin{array}{c}\text { Men } \\
\text { THC: } 2.5 \\
\text { 11-OH-THC: } 2.0\end{array}$ & \multirow{4}{*}{ Effects were not assessed. } \\
\hline & & & & & 15 mg THC (women) & $\begin{array}{c}\text { Women } \\
\text { THC: } 9.4 \pm 4.5^{\mathrm{b}} \\
11-\mathrm{OH}-\mathrm{THC}: 5.9 \pm 2.8^{\mathrm{b}}\end{array}$ & $\begin{array}{c}\text { Women } \\
\text { THC: } 1.75 \\
\text { 11-OH-THC: } 1.75\end{array}$ & \\
\hline & & & \multirow{2}{*}{$\begin{array}{l}\text { Intravenous } \\
\text { (infusion pump) }\end{array}$} & \multirow{2}{*}{$\begin{array}{l}\text { Human serum } \\
\text { albumin }\end{array}$} & 4 mg THC (men) & $\begin{array}{c}\text { Men } \\
\text { THC: } 71 \pm 34^{\mathrm{b}} \\
11-\mathrm{OH}-\mathrm{THC}: 3.7 \pm 2.3^{\mathrm{b}}\end{array}$ & $\begin{array}{c}\text { Men } \\
\text { THC: } 0.42 \\
\text { 11-OH-THC: } 0.5\end{array}$ & \\
\hline & & & & & $2.2 \mathrm{mg} \mathrm{THC} \mathrm{(women)}$ & $\begin{array}{c}\text { Women } \\
\text { THC: } 85 \pm 26^{\mathrm{b}} \\
11-\mathrm{OH}-\mathrm{THC}: 3.8 \pm 2.8^{\mathrm{b}}\end{array}$ & $\begin{array}{c}\text { Women } \\
\text { THC: } 0.17 \\
\text { 11-OH-THC: } 0.33 \\
\end{array}$ & \\
\hline \multirow{2}{*}{ Haney et al., 2003 [38] } & \multirow{2}{*}{$R, D B, P, C$} & \multirow{2}{*}{$\begin{array}{c}7 \mathrm{M} \\
\text { Cannabis smokers }\end{array}$} & Oral & $\begin{array}{l}\text { Capsules } \\
\left(\text { Marinol }^{\mathbb{R}}\right)\end{array}$ & $\begin{array}{l}30 \mathrm{mg} \text { THC29, } \\
\text { Placebo naltrexone }\end{array}$ & $\begin{array}{c}\text { THC: } 29.9 \pm 9.5^{\mathrm{b}} \\
\text { THC-COOH: } 121.9 \pm 43.5^{\mathrm{b}}\end{array}$ & $\begin{array}{c}\text { THC: } 4^{\mathrm{b}} \\
\text { THC-COOH: } 2^{\text {b }}\end{array}$ & \multirow{2}{*}{$\begin{array}{c}\text { Subjective effects } \\
\text { Increase in ratings of Good Drug Effect, High, } \\
\text { and Stimulated. Vital effects Decreased HR. } \\
\text { Worsened psychomotor performance. }\end{array}$} \\
\hline & & & Oral & $\begin{array}{c}\text { Capsules } \\
\left.\text { (Marinol }^{\mathbb{R}}\right)\end{array}$ & $\begin{array}{c}30 \mathrm{mg} \text { THC } \\
50 \mathrm{mg} \text { naltrexone }\end{array}$ & $\begin{array}{c}\text { THC: } 21.2 \pm 8.6^{\mathrm{b}} \\
\text { THC-COOH: } 139.0 \pm 36.2^{\mathrm{b}}\end{array}$ & $\begin{array}{c}\text { THC: } 2^{\text {b }} \\
\text { THC-COOH: } 3^{\text {b }}\end{array}$ & \\
\hline \multirow{4}{*}{ Naef et al., 2003 [39] } & \multirow{4}{*}{$R, D B, P, C$} & \multirow{4}{*}{$\begin{array}{c}6 \mathrm{M}, 6 \mathrm{~F} \\
\text { Cannabis naive }\end{array}$} & Oral & $\begin{array}{l}\text { Capsules } \\
\left.\text { (Marinol }{ }^{\mathbb{Q}}\right)\end{array}$ & $20 \mathrm{mg}$ THC & $\begin{array}{c}\text { THC: } 7.2 \pm 6.9^{\mathrm{b}, \mathrm{e}} \\
\text { 11-OH-THC: } 19.7 \pm 6.9^{\mathrm{b}, \mathrm{e}} \\
\text { 11-COOH-THC: } 241.4 \pm 73 . \mathrm{b}, \mathrm{e}\end{array}$ & $\begin{array}{c}\text { THC: } 1-2^{\mathrm{b}, \mathrm{e}} \\
\text { 11-OH-THC: } 2^{\mathrm{b}, \mathrm{e}} \\
\text { 11-COOH-THC: } 2^{\mathrm{b}} 4^{\mathrm{b}, \mathrm{e}}\end{array}$ & \multirow{4}{*}{$\begin{array}{c}\text { Subjective effects } \\
\text { Psychotropic and somatic side-effects were } \\
\text { common but usually mild. } \\
\text { Paint tests } \\
\text { No significant reduction in pain. }\end{array}$} \\
\hline & & & Oral & Capsules & $\begin{array}{c}20 \mathrm{mg} \mathrm{THC} \\
30 \mathrm{mg} \text { morphine } \mathrm{HCl}\end{array}$ & $\begin{array}{c}\text { THC: } 6.7 \pm 7.3^{\mathrm{b}, \mathrm{e}} \\
\text { 11-OH-THC: } 7.9 \pm 8.3^{\mathrm{b}, \mathrm{e}} \\
11-C O O H-T H C: 134.7 \pm 65.12^{\mathrm{b}, \mathrm{e}}\end{array}$ & $\begin{array}{l}- \\
- \\
-\end{array}$ & \\
\hline & & & Oral & $\begin{array}{l}\text { Capsules } \\
\text { (placebo) }\end{array}$ & 30 mg morphine $\mathrm{HCl}$ & & & \\
\hline & & & Oral & $\begin{array}{l}\text { Capsules } \\
\text { (placebo) }\end{array}$ & & & & \\
\hline \multirow{4}{*}{ Guy et al., 2004 [40] } & \multirow{4}{*}{$\begin{array}{l}\text { R, OL, C } \\
\text { followed by an } \\
\text { NR oral dose }\end{array}$} & \multirow{4}{*}{$\begin{array}{l}6 \mathrm{M}, 6 \mathrm{~F} \\
\begin{array}{l}\text { Previous experience of } \\
\text { cannabis use }\end{array}\end{array}$} & Oral & Capsules & $\begin{array}{l}10 \mathrm{mg} \mathrm{THC} \\
10 \mathrm{mg} \text { CBD }\end{array}$ & $\begin{array}{c}\text { THC: } 6.35 \pm 3.12(3.04-4.55) \\
\text { CBD: } 2.47 \pm 2.23(0.47-7.55) \\
11-\mathrm{OH}-\mathrm{THC}: 7.87 \pm 2.96 \\
(4.79-13.64)\end{array}$ & $\begin{array}{c}\text { THC: } 1.05 \pm 0.65(0.5-2.75) \\
\text { CBD: } 1.27 \pm 0.84(0.5-3) \\
11-\mathrm{OH}-\mathrm{THC}: 1.36 \pm 0.63 \\
(0.75-3)\end{array}$ & \multirow{4}{*}{ Effects were not assessed. } \\
\hline & & & Sublingual & $\begin{array}{l}\text { Liquid spray } \\
\text { (Sativex }^{\circledR} \text { ) }\end{array}$ & $\begin{array}{l}10 \mathrm{mg} \mathrm{THC} \\
10 \mathrm{mg} \text { CBD }\end{array}$ & $\begin{array}{c}\text { THC: } 5.54 \pm 3.35(1.14-12.13) \\
\text { CBD: } 2.50 \pm 1.83(0.27-6.55) \\
11-O H-T H C: 6.24 \pm 2.74 \\
(2.67-10.77)\end{array}$ & $\begin{array}{c}\text { THC: } 1.63 \pm 0.59(1-3) \\
\text { CBD: } 1.63 \pm 0.68(0.75-3) \\
\text { 11-OH-THC: } 1.58 \pm 0.44 \\
(1-2.75)\end{array}$ & \\
\hline & & & Buccal & $\begin{array}{l}\text { Liquid spray } \\
\left(\text { (Sativex }{ }^{\circledR}\right)\end{array}$ & $\begin{array}{l}10 \mathrm{mg} \text { THC } \\
10 \mathrm{mg} \text { CBD }\end{array}$ & $\begin{array}{c}\text { THC: } 6.14 \pm 5.37(0.88-19.78) \\
\text { CBD: } 3.02 \pm 3.15(0.29-9.91) \\
11-O H-T H C: 6.13 \pm 2.88 \\
(1.83-11.25)\end{array}$ & $\begin{array}{c}\text { THC: } 2.40 \pm 1.08(1-4.5) \\
\text { CBD: } 2.78 \pm 1.31(1-4.5) \\
11-\mathrm{OH}-\mathrm{THC}: 2.40 \pm 1.17(1-4.5)\end{array}$ & \\
\hline & & & Oro-pharyngeal & $\begin{array}{l}\text { Liquid spray } \\
\left.\text { (Sativex }^{\circledR}\right)\end{array}$ & $\begin{array}{l}10 \mathrm{mg} \text { THC } \\
10 \mathrm{mg} \text { CBD }\end{array}$ & $\begin{array}{c}\text { THC: } 6.11 \pm 4.00(1.94-15.68) \\
\text { CBD: } 2.61 \pm 1.91(0.41-6.36) \\
11-\mathrm{OH}-\mathrm{THC}: 6.45 \pm 2.91 \\
(2.95-13.49)\end{array}$ & $\begin{array}{c}\text { THC: } 2.23 \pm 1.52(0.75-5) \\
\text { CBD: } 2.04 \pm 1.13(0.75-5) \\
11-\mathrm{OH}-\mathrm{THC}: 2.40 \pm 1.22 \\
(1.25-5)\end{array}$ & \\
\hline $\begin{array}{l}\text { Menetrey et al., } \\
2005 \text { [41] }\end{array}$ & & & & Table 2 for $\mathrm{r}$ & on capsules and dec & tion administration. & & \\
\hline
\end{tabular}


Table 1. Cont.

\begin{tabular}{|c|c|c|c|c|c|c|c|c|}
\hline References & Study Design & Participants & $\begin{array}{c}\text { Route of } \\
\text { Administration }\end{array}$ & Formulation & Dose & $\begin{array}{c}\text { Cmax (ng/mL) } \\
\text { Mean } \pm \text { SD, Median (Range) }\end{array}$ & $\operatorname{Tmax}(\mathrm{h})$ & Pharmacological Effects \\
\hline \multirow{3}{*}{$\begin{array}{l}\text { Nadulski et al., } \\
\quad 2005 \text { [42] }\end{array}$} & \multirow{3}{*}{ DB, P, C } & \multirow{3}{*}{24} & Oral & Capsules & $\begin{array}{l}10 \mathrm{mg} \text { THC } \\
5.4 \mathrm{mg} \text { CBD }\end{array}$ & $\begin{array}{c}\text { THC: } 4.05(1.18-10.27) \\
\text { CDD: } 0.95 \text { (0.30-2.57) } \\
\text { 11-OH-THC: } 4.88(1.83-12.34) \\
\text { THC-COOH:35.46 (19.2-70.6) }\end{array}$ & $\begin{array}{c}\text { THC: } 0.93(0.55-2.08) \\
\text { CBD: } 0.99(0.5-2) \\
\text { 11-OH-THC: } 1.67(0.62-2.17) \\
\text { THC-COOH: } 1.92(1.08-3.83)\end{array}$ & \multirow{3}{*}{ Effects were not assessed. } \\
\hline & & & Oral & Capsules & $10 \mathrm{mg}$ THC & $\begin{array}{c}\text { THC: } 3.20(0.67-7.99) \\
\text { 11-OH-THC: } 4.48(1.12-11.14) \\
\text { THC-COOH: } 32.9(12.03-57.63)\end{array}$ & $\begin{array}{c}\text { THC: } 1.06(0.5-3.05) \\
\text { 11-OH-THC: } 1.5(0.5-3.17) \\
\text { THC-COOH: } 2.07(0.62-3.92)\end{array}$ & \\
\hline & & & Oral & Capsules & Placebo & & & \\
\hline $\begin{array}{l}\text { Goodwin et al., } \\
2006[43]\end{array}$ & \multicolumn{7}{|c|}{ See also Table 2 for results on capsules and oil administration. } & \\
\hline $\begin{array}{l}\text { Schwilke et al., } \\
2009 \text { [44] }\end{array}$ & $\mathrm{NR}, \mathrm{OL}, \mathrm{NP}, \mathrm{MD}$ & $\begin{array}{l}\text { 6 M Daily smokers (positive in } \\
\text { cannabinoids, smoked within } \\
\text { the previous } 24 \mathrm{~h} \text { ) }\end{array}$ & Oral & $\begin{array}{c}\text { Capsules } \\
\text { (Marinol }^{\mathbb{B}} \text { ) }\end{array}$ & $\begin{array}{c}\text { Escalating total daily } \\
\text { doses (40-120 mg) for } \\
7 \text { days } \\
\text { First dose } 20 \mathrm{mg} \text { THC }\end{array}$ & $\begin{array}{c}\text { After 1st dose (single dose): } \\
\text { THC: } 12.4 \pm 3.4 \\
\text { 11-OH-THC: } 8.2 \pm 2.0 \\
\text { THC-COOH: } 75.8 \pm 9.4\end{array}$ & $\begin{array}{c}\text { After 1st dose: } \\
\text { THC: } 2.8(0.33) \\
\text { 11-OH-THC: } 2.5(0.18) \\
\text { THC-COOH: } 3.3(0.56)\end{array}$ & Effects were not assessed. \\
\hline \multirow{4}{*}{$\begin{array}{l}\text { Karschner et al., } \\
\quad 2011[45]\end{array}$} & \multirow{4}{*}{$\mathrm{R}, \mathrm{DB}, \mathrm{P}, \mathrm{DD}$} & \multirow{4}{*}{$\begin{array}{c}6 \mathrm{M}, 3 \mathrm{~F} \\
\text { Cannabis smokers }\end{array}$} & Oral & $\begin{array}{l}\text { Capsules } \\
\text { (dronabinol) }\end{array}$ & $5 \mathrm{mg}$ THC & $\begin{array}{c}\text { THC: } 4.7 \pm 0.9,4.6(1.4-10.4) \\
11-\mathrm{OH}-\mathrm{THC}: 3.0 \pm 0.4,2.6 \\
(1.8-5.9) \\
\text { THC-COOH: } 69.3 \pm 17.6,57.1 \\
(15.9-179.7)\end{array}$ & $\begin{array}{c}\text { THC: } 3.2 \pm 0.3,3.1(1.5-4.5) \\
11-\mathrm{OH}-\mathrm{THC}: 3.3 \pm 0.4,3.3 \\
\text { (1.5-5.6) } \\
\text { THC-COOH: } 4.4 \pm 0.5,4.3 \\
(2.7-7.5)\end{array}$ & \multirow{4}{*}{ Effects were not assessed. } \\
\hline & & & Oral & $\begin{array}{c}\text { Capsules } \\
\text { (dronabinol) }\end{array}$ & $15 \mathrm{mg} \mathrm{THC}$ & $\begin{array}{c}\text { THC: } 14.3 \pm 2.7,11.2(3.3-28.5) \\
\text { 11-OH-THC: } 111.1 \pm 2.0,9.3 \\
\text { (3.6-19.5) } \\
\text { THC-COOH: } 133.6 \pm 36.3,102.1 \\
(44.5-409.0) \\
\end{array}$ & $\begin{array}{c}\text { THC: } 3.4 \pm 0.5,3.4(1.2-5.5) \\
11-\mathrm{OH}-\mathrm{THC}: 3.4 \pm 0.4,3.6 \\
(1.0-5.5) \\
\text { THC-COOH: } 4.9 \pm 0.5,5.5 \\
(2.4-7.5)\end{array}$ & \\
\hline & & & Sublingual & Spray $\left(\right.$ Sativex $\left.{ }^{\circledR}\right)$ & $\begin{array}{l}5.4 \mathrm{mg} \mathrm{THC} \\
5.0 \mathrm{mg} \text { CBD }\end{array}$ & $\begin{array}{c}\text { THC: } 5.1 \pm 1.0,5.1(1.2-9.6) \\
\text { CBD } 1.6 \pm 0.4,1.2(0.6-3.9) \\
11-\mathrm{OH}-\mathrm{HC}: 4.2 \pm 0.7,3.7 \\
(2.1-7.5) \\
\text { THC-COOH: } 108.0 \pm 30.5,79.8 \\
(19.1-281.6) \\
\end{array}$ & $\begin{array}{c}\text { THC: } 3.3 \pm 0.3,3.5(1.2-4.5) \\
\text { CBD: } 3.7 \pm 0.5,3.6(1.0-5.5) \\
11-\mathrm{OH}-\mathrm{THC}: 3.6 \pm 0.6,3.3 \\
(1.0-7.5) \\
\text { THC-COOH: } 4.4 \pm 0.7,4.5 \\
(1.2-7.5)\end{array}$ & \\
\hline & & & Sublingual & Spray $\left(\right.$ Sativex $\left.{ }^{\circledR}\right)$ & $\begin{array}{l}16.2 \mathrm{mg} \text { THC } \\
15.0 \mathrm{mg} \text { CBD }\end{array}$ & $\begin{array}{c}\text { THC: } 15.3 \pm 3.4,14.5(3.2-38.2) \\
\text { CDD: } 6.7 \pm 2.0,3.7(2.0-20.5) \\
11-\mathrm{OH}-\mathrm{THC}: 8.4 \pm 1.2,7.6 \\
(3.8-13.7) \\
\text { THC-COOH: } 126.6 \pm 25.9,92.4 \\
(55.9-304.1)\end{array}$ & $\begin{array}{c}\text { THC: } 4.0 \pm 0.5,4.5(1.2-5.6) \\
\text { CBD: } 4.0 \pm 0.5,4.5(1.2-5.6) \\
11-\mathrm{OH}-\mathrm{THC}: 3.9 \pm 0.5,3.7 \\
(1.2-5.6) \\
\text { THC-COOH: } 4.8 \pm 0.3,5.0 \\
(2.6-5.6)\end{array}$ & \\
\hline $\begin{array}{l}\text { Karschner et al., } \\
\quad 2012 \text { [46] }\end{array}$ & $\mathrm{NR}, \mathrm{OL}, \mathrm{NP}, \mathrm{MD}$ & $\begin{array}{l}10 \mathrm{M} \\
\text { Daily smokers (positive } \\
\text { cannabinoids, smoked within } \\
\text { the previous } 24 \mathrm{~h} \text { ) }\end{array}$ & Oral & $\begin{array}{c}\text { Capsules } \\
\text { (Marinol }^{\mathbb{\circledR}} \text { ) }\end{array}$ & $\begin{array}{c}\text { Escalating total daily } \\
\text { doses }(40-120 \mathrm{mg}) \text { for } \\
7 \text { days } \\
\text { Each dose of } 20 \mathrm{mg} \\
\text { THC }\end{array}$ & $\begin{array}{c}\text { After 1st dose (single dose): } \\
\text { THC : } 8.7 \pm \pm .8,6.4(4.1-17.5) \\
11-\mathrm{OH}-\mathrm{THC}: 4.0 \pm 2.1,3.4 \\
(1.8-7.8) \\
\text { THC-COOH: } 78.4 \pm 15.9,36.6 \\
(19.7-68.7) \\
\end{array}$ & $\begin{array}{c}\text { After 1st dose: } \\
\text { THC: } 3.0 \pm 0.9,3.0(2.0-4.0) \\
11-\mathrm{OH}-\mathrm{THC}: 2.8 \pm 0.9,3.0 \\
(2.0-5.0) \\
\text { THC-COOH: } 3.1 \pm 1.0,3.0 \\
(2.0-5.0)\end{array}$ & Effects were not assessed. \\
\hline \multirow{3}{*}{$\begin{array}{l}\text { Martin-santos et al., } \\
2012[47]\end{array}$} & \multirow{3}{*}{$\mathrm{R}, \mathrm{DB}, \mathrm{P}, \mathrm{C}$} & \multirow{3}{*}{$\begin{array}{c}16 \mathrm{M} \\
\text { Previous experience of } \\
\text { cannabis use (less than } 15 \\
\text { times in their lifetime) }\end{array}$} & Oral & Capsules & $10 \mathrm{mg}$ THC & $\begin{array}{l}\text { THC: } 0.67 \pm 0.66^{\mathrm{b}} \\
\text { THC-COOH: } \approx 5.6^{\mathrm{b}, \mathrm{d}} \\
11-\mathrm{OH}-\mathrm{THC}: \approx 0.73^{\mathrm{b}, \mathrm{d}}\end{array}$ & THC: $2 \mathrm{~h}^{\mathrm{b}}$ & \multirow{3}{*}{$\begin{array}{c}\text { Subjective effects } \\
\text { Signnificant changes in PANSS, anxiety } \\
\text { (STAI-S), dysphoria (ARCI), sedatation (VAMS, } \\
\text { ARCI), and the level of subjective intoxication } \\
\text { (ASI, ARCI) Vital effects significant increase in } \\
\text { HR No significant differences in SBP and DBP. }\end{array}$} \\
\hline & & & Oral & Capsules & $600 \mathrm{mg}$ CBD & & & \\
\hline & & & Oral & $\begin{array}{l}\text { Capsules } \\
\text { (placebo) }\end{array}$ & & & & \\
\hline
\end{tabular}


Table 1. Cont.

\begin{tabular}{|c|c|c|c|c|c|c|c|c|}
\hline References & Study Design & Participants & $\begin{array}{c}\text { Route of } \\
\text { Administration } \\
\end{array}$ & Formulation & Dose & $\begin{array}{c}\mathrm{Cmax}(\mathrm{ng} / \mathrm{mL}) \\
\text { Mean } \pm \mathrm{SD} \text {, Median (Range) }\end{array}$ & $\operatorname{Tmax}(\mathrm{h})$ & Pharmacological Effects \\
\hline \multirow{3}{*}{ Eichler et al., 2012 [48] } & \multirow{3}{*}{$\mathrm{R}, \mathrm{DB}, \mathrm{C}$} & \multirow{3}{*}{$\begin{array}{c}9 \mathrm{M} \\
\text { Non smokers }\end{array}$} & Oral & $\begin{array}{l}\text { Capsules } \\
\left(\text { Marinol }^{\mathbb{R}}\right)\end{array}$ & $20 \mathrm{mg}$ THC & $\begin{array}{c}\text { THC: } 1.03 \pm 1.65,0.48^{\mathrm{e}, \mathrm{g}} \\
\text { CBD: } 0.00 \pm 0.00,0.0^{\mathrm{e}, \mathrm{g}} \\
\text { 11-OH-THC: } 0.99 \pm 0.63,0.84 \mathrm{e}, \mathrm{g} \\
\text { THC-COOH: } 7.13 \pm 5.64,7.61^{\mathrm{e}, \mathrm{g}} \\
\text { CBN: } 0.64 \pm 0.72,0.37^{\mathrm{e}, \mathrm{g}}\end{array}$ & $\begin{array}{c}\text { THC: } 1.06 \pm 0.19,1.0^{\mathrm{e}} \\
\text { CBD: NA } \\
11-\mathrm{OH}-\mathrm{THC}: 1.67 \pm 0.51,2 . \mathrm{e}^{\mathrm{e}} \\
\text { THC-COOH: } 1.78 \pm 0.96,2.0^{\mathrm{e}} \\
\text { CBN: } 1.06 \pm 0.57,1.0^{\mathrm{e}}\end{array}$ & \multirow{3}{*}{$\begin{array}{c}\text { Subjective effects } \\
\text { Mild psychotropic effects, with no significant } \\
\text { differences between treatments. }\end{array}$} \\
\hline & & & Oral & $\begin{array}{l}\text { Capsules } \\
\text { (extract from } \\
\text { heated Herba } \\
\text { Cannabis) }\end{array}$ & $\begin{array}{l}17.6 \mathrm{mg} \mathrm{THC} \\
27.8 \mathrm{mg} \text { CBD }\end{array}$ & $\begin{array}{c}\text { THC: } 0.42 \pm 0.39,0.25^{\mathrm{e}, \mathrm{g}} \\
\text { CBD: } 0.30 \pm 0.21,0.27^{\mathrm{e}, \mathrm{g}} \\
\text { 11-OH-THC: } 0.73 \pm 0.69,0.50^{\mathrm{e}, \mathrm{g}} \\
\text { THC-COOH: } 5.81 \pm 7.59,3.46^{\mathrm{e}, \mathrm{g}} \\
\text { CBN: } 0.60 \pm 0.36,0.56^{\mathrm{e}, \mathrm{g}}\end{array}$ & $\begin{array}{c}\text { THC: } 0.78 \pm 0.27,1.0^{\mathrm{e}} \\
\text { CBD } 0.83 \pm 0.51,0 . \mathrm{e}^{\mathrm{e}} \\
\text { 11-OH-THC: } 1.44 \pm 0.69,2.0^{\mathrm{e}} \\
\text { THC-COOH: } 2.89 \pm 1.05,2.0^{\mathrm{e}} \\
\text { CBN: } 0.94 \pm 0.45,1.0^{\mathrm{e}}\end{array}$ & \\
\hline & & & Oral & $\begin{array}{c}\text { Capsules } \\
\text { (extract from } \\
\text { unheated Herba } \\
\text { Cannabis) } \\
\end{array}$ & $\begin{array}{l}10.4 \mathrm{mg} \mathrm{THC} \\
14.8 \mathrm{mg} \text { CBD }\end{array}$ & $\begin{array}{c}\text { THC: } 1.02 \pm 0.78,0.71^{\mathrm{e}, \mathrm{g}} \\
\text { CBD: } 1.24 \pm 0.87,0.96^{\mathrm{e}, \mathrm{g}} \\
\text { 11-OH-THC: } 0.57 \pm 0.42,0.50^{\mathrm{e}, \mathrm{g}} \\
\text { THC-COOH: } 1.94 \pm 1.11,2.28^{\mathrm{e}, \mathrm{g}} \\
\text { CBN: } 0.54 \pm 0.30,0.58^{\mathrm{e}, \mathrm{g}}\end{array}$ & $\begin{array}{c}\text { THC: } 1.17 \pm 0.66,1.0^{\mathrm{e}} \\
\text { CBD: } 1.17 \pm 1.17,1.0^{\mathrm{e}} \\
11-O H-T H C: 1.00 \pm 0.42,1.0^{\mathrm{e}} \\
\text { THC-COOH: } 2.11 \pm 0.78,2.0^{\mathrm{e}} \\
\text { CBN: } 1.00 \pm 0.42,1.0^{\mathrm{e}} \\
\end{array}$ & \\
\hline \multirow{7}{*}{ Lile JA et al., 2013 [49] } & \multirow{7}{*}{$B, P, C$} & \multirow{7}{*}{$\begin{array}{l}4 \mathrm{M}, 3 \mathrm{~F} \\
\text { Only } 5 \text { completed all doses } \\
\text { Regular cannabis use }\end{array}$} & Oral & $\begin{array}{c}\text { Capsules } \\
\left(\text { Marinol }{ }^{\mathbb{R}}\right)\end{array}$ & $15 \mathrm{mg}$ THC & $\begin{array}{c}\text { THC: } \approx 5^{\mathrm{d}} \\
11-\mathrm{OH}-\mathrm{THC}: \approx 2-3^{\mathrm{d}}\end{array}$ & $\begin{array}{c}\text { THC: } 3^{\mathrm{d}} \\
\text { 11-OH-THC: } 3^{\mathrm{d}}\end{array}$ & \multirow{7}{*}{$\begin{array}{c}\text { Vital effects } \\
\text { Increase in HR. } \\
\text { SBP decreased after } 30 \mathrm{mg} \text { dose but increased } \\
\text { after } 75 \text { and } 90 \mathrm{mg} \text { doses. } \\
\text { No changes in DBP. } \\
\text { Decrease in finger temperature. } \\
\text { Psychomotor performance } \\
\text { Worsened psychomotor performance. }\end{array}$} \\
\hline & & & Oral & $\begin{array}{c}\text { Capsules } \\
\text { (Marinol }^{\mathbb{B}} \text { ) }\end{array}$ & 30 mg THC & $\begin{array}{c}\text { THC: } \approx 10 \mathrm{~d} \\
11-\mathrm{OH}-\mathrm{THC}: \approx 5^{\mathrm{d}}\end{array}$ & $\begin{array}{c}\text { THC: } 3^{\mathrm{d}} \\
\text { 11-OH-THC: } 3^{\mathrm{d}}\end{array}$ & \\
\hline & & & Oral & $\begin{array}{c}\text { Capsules } \\
\left.\text { (Marinol }^{\mathbb{B}}\right)\end{array}$ & $45 \mathrm{mg}$ THC & $\begin{array}{c}\text { THC: } \approx 17-18^{\mathrm{d}} \\
\text { 11-OH-THC: } \approx 8-9 \mathrm{~d}\end{array}$ & $\begin{array}{c}\text { THC: } 2.5^{\mathrm{d}} \\
11-\mathrm{OH}-\mathrm{THC}: 2^{\mathrm{d}}\end{array}$ & \\
\hline & & & Oral & $\begin{array}{c}\text { Capsules } \\
\left.\text { (Marinol }^{\circledR}\right)\end{array}$ & $60 \mathrm{mg}$ THC & $\begin{array}{c}\text { THC: } \approx 45 \mathrm{~d} \\
11-\mathrm{OH}-\mathrm{THC}: \approx 11^{\mathrm{d}}\end{array}$ & $\begin{array}{c}\text { THC: } 3.5^{\mathrm{d}} \\
\text { 11-OH-THC: } 3^{\mathrm{d}}\end{array}$ & \\
\hline & & & Oral & $\begin{array}{l}\text { Capsules } \\
\left(\text { Marinol }^{\mathbb{B}}\right)\end{array}$ & 75 mg THC & $\begin{array}{c}\text { THC: } \approx 42-43 \mathrm{~d} \\
11-\mathrm{OH}-\mathrm{THC}: \approx 12-13^{\mathrm{d}}\end{array}$ & $\begin{array}{c}\text { THC: } 4^{\mathrm{d}} \\
\text { 11-OH-THC: } 4^{\mathrm{d}}\end{array}$ & \\
\hline & & & Oral & $\begin{array}{c}\text { Capsules } \\
\left(\text { Marinol }^{\mathbb{B}}\right)\end{array}$ & $90 \mathrm{mg}$ THC & $\begin{array}{c}\text { THC: } \approx 53 \mathrm{~d} \\
11-\mathrm{OH}-\mathrm{THC}: \approx 20^{\mathrm{d}}\end{array}$ & $\begin{array}{c}\text { THC: } 4 \mathrm{~d} \\
\text { 11-OH-THC: } 4^{\mathrm{d}}\end{array}$ & \\
\hline & & & Oral & $\begin{array}{l}\text { Capsules } \\
\text { (placebo) }\end{array}$ & & & & \\
\hline \multirow{2}{*}{ Parikh et al., 2016 [50] } & \multirow{2}{*}{$\mathrm{R}, \mathrm{OL}, \mathrm{C}$} & \multirow{2}{*}{$\begin{array}{l}51 \mathrm{MF} \\
\text { No cannabis use in the } \\
\text { previous } 90 \text { days }\end{array}$} & Oral & $\begin{array}{l}\text { Oral solution } \\
\text { (Dronabinol) }\end{array}$ & $4.25 \mathrm{mg}$ THC & $\begin{array}{c}\text { THC Replicate 1: } 1.81 \pm 1.26 \\
\text { THC Replicate 2: } 2.08 \pm 1.30 \\
\text { 11-OH-THC Replicate 1: } \\
\text { 2.53 } 1.38 \\
\text { 11-OH-THC Replicate 2: } \\
\text { 3.01 } 1.56 \\
\end{array}$ & $\begin{array}{c}\text { THC Replicate 1: } 1.50 \\
(0.50-4.00) \\
\text { THC Replicate 2: } 1.00 \\
(0.50-3.02) \\
\text { 11-OH-THC Replicate 1: } 1.50 \\
(0.75-4.00) \\
\text { 11-OH-THC Replicate 2: } 1.50 \\
(0.50-3.02) \\
\end{array}$ & \multirow{2}{*}{ Effects were not assessed. } \\
\hline & & & Oral & $\begin{array}{c}\text { Capsules } \\
\text { (Dronabinol) }\end{array}$ & $5 \mathrm{mg}$ THC & $\begin{array}{c}\text { THC Replicate 1: } 2.20 \pm 1.51 \\
\text { THC Replicate 2: } 2.61 \pm 1.69 \\
\text { 11-OH-THC Replicate: } 3.28 \pm \\
\text { 1.78 11-11-OH-THC Replicate 2: } \\
3.98 \pm 2.51\end{array}$ & $\begin{array}{c}\text { THC Replicate 1: } 1.00 \\
(0.50-6.00) \\
\text { THC Replicate 2: } 1.50 \\
(0.50-6.00) \\
\text { 11-OH-THC Replicate 1: } 1.60 \\
(0.75-6.00) \\
\text { 11-OH-THC Replicate 2: } 1.50 \\
(0.50-6.00)\end{array}$ & \\
\hline
\end{tabular}


Table 1. Cont.

\begin{tabular}{|c|c|c|c|c|c|c|c|c|}
\hline References & Study Design & Participants & $\begin{array}{c}\text { Route of } \\
\text { Administration }\end{array}$ & Formulation & Dose & $\begin{array}{c}\text { Cmax }(\mathrm{ng} / \mathrm{mL}) \\
\text { Mean } \pm \mathrm{SD}, \text { Median (Range) }\end{array}$ & $\operatorname{Tmax}(\mathrm{h})$ & Pharmacological Effects \\
\hline \multirow{2}{*}{$\begin{array}{l}\text { Cherniakov et al., } \\
\quad 2017[51]\end{array}$} & \multirow[b]{2}{*}{$\mathrm{OL}, \mathrm{C}$} & \multirow{2}{*}{$\begin{array}{l}9 \mathrm{M} \\
\text { Not exposed within the } \\
\text { previous } 4 \text { weeks }\end{array}$} & Sublingual & Spray $\left(\right.$ Sativex $\left.{ }^{\circledR}\right)$ & $\begin{array}{l}10.8 \mathrm{mg} \text { THC } \\
10.0 \mathrm{mg} \text { CBD }\end{array}$ & $\begin{array}{l}\text { THC: } 1.8 \pm 0.2 \\
\text { CBD: } 0.5 \pm 0.1\end{array}$ & $\begin{array}{l}\text { THC: } 2(1-4) \\
\text { CBD: } 3(1-5)\end{array}$ & \multirow[b]{2}{*}{ Effects were not assessed } \\
\hline & & & Oral & $\begin{array}{l}\text { THC-CBD-piperine } \\
\text {-PNL capsule }\end{array}$ & $\begin{array}{l}10.8 \mathrm{mg} \mathrm{THC} \\
10.0 \mathrm{mg} \text { CBD }\end{array}$ & $\begin{array}{l}\text { THC: } 5.4 \pm 0.01 \\
\text { CBD: } 2.1 \pm 0.4\end{array}$ & $\begin{array}{l}\text { THC: } 1(1-1.5) \\
\text { CBD: } 1(0.5-1.5)\end{array}$ & \\
\hline
\end{tabular}

Abbreviations: Cmax, maximum concentration after administration; SD, standard deviations; R, randomized; NR, not-randomized; OL, open label; DB, double-blind; B, blind; P, placebo-controlled; NP, not placebo-controlled; C, crossover; DD, double-dummy; MD, multiple dose; M, male; F, female; THC, $\Delta-9$ tetrahydrocannabinol; THCA, $\Delta$-9-tetrahydrocannabinolic acid A; CBD, cannabidiol; CBDA, cannabidiolic acid; 11-OH-THC, 11-hydroxy-THC; THC-COOH, 11-nor-9-carboxy-THC; THC-COOH-gluc, THC-COOH-glucuronide; THCV-COOH, 11-nor-9-carboxy-tetrahydrocannabivarin; BP, blood pressure; SBP, systolic blood pressure; DBP, diastolic blood pressure; HR, heart rate; ARCI, Addiction Research Center Inventory; VAS, visual analogue scales; VAMS, visual analogue mood scale; PANSS, Positive And Negative Symptom Scale; STAI, State-Trait Anxiety Inventory; ASI, Addiction Severity Index. a Range corresponds to the range of Cmax. ${ }^{b}$ The maximum value of the time-course of plasma THC. ${ }^{c}$, the median instead of mean. ${ }^{d}$, data deduced from a figure. ${ }^{e}$, the original values for values corresponds to the range of Cmax. ${ }^{b}$ The maximum value of the time-course of plasma THC. ${ }^{c}$, the median instead of mean. ${ }^{d}$, data deduced from a figure. ${ }^{e}$, the original values for values
presented as the standard error or coefficient of variation of the mean have been transformed in the table to standard deviation. ${ }^{\mathrm{f}}$, the mean values have been calculated from the values reported in the article. ${ }^{g}$, values converted from $\mathrm{pmol} / \mathrm{mL}$ to $\mathrm{ng} / \mathrm{mL}$

Table 2. Summary of studies included in this systematic review reporting the pharmacokinetic parameters for THC following oil or decoction administration

\begin{tabular}{|c|c|c|c|c|c|c|c|c|}
\hline References & Study Design & Participants & $\begin{array}{c}\text { Route of } \\
\text { Administration }\end{array}$ & Formulation & Dose & $\begin{array}{c}\text { Cmax (ng/mL) } \\
\text { Mean } \pm \text { SD, Median (Range) }\end{array}$ & $\operatorname{Tmax}(\mathrm{h})$ & Pharmacological Effects \\
\hline \multirow{5}{*}{$\begin{array}{l}\text { Menetrey et al., } \\
2005[41]\end{array}$} & \multirow{5}{*}{$\mathrm{R}, \mathrm{DB}, \mathrm{P}, \mathrm{C}$} & \multirow{5}{*}{$\begin{array}{c}8 \mathrm{M} \\
\text { Occasional cannabis smokers }\end{array}$} & Oral & Milk decoction & $16.5 \mathrm{mg}$ THC & $\begin{array}{c}\text { THC: } 3.8(1.5-8.3) \mathrm{b} \\
\text { 11-OH-THC: } 4.7(2.9-7.0) \mathrm{b} \\
\text { THC-COOH: } 27.8(14.1-42.4) \mathrm{b}\end{array}$ & $\begin{array}{c}\text { THC: } 1 \mathrm{~b} \\
\text { 11-OH-THC: } 1 \mathrm{~b} \\
\text { THC-COOH: } 4 \mathrm{~b}\end{array}$ & \multirow{5}{*}{$\begin{array}{c}\text { Subjective effects } \\
\text { Prototypical effects of THC with a strong } \\
\text { feeling of highness. } \\
\text { Vital effects } \\
\text { Slight to moderate conjunctival reddening. } \\
\text { Slight to moderate tachycardia } \\
\text { Increase of HR after decoction. }\end{array}$} \\
\hline & & & Oral & Milk decoction & $45.7 \mathrm{mg}$ THC & $\begin{array}{c}\text { THC: } 8.4(3.9-13.1) \mathrm{b} \\
\text { 11-OH-THC: } 12.8(3.4-24.7) \mathrm{b} \\
\text { THC-COOH: } 66.2(29.0-99) \mathrm{b}\end{array}$ & $\begin{array}{c}\text { THC: } 1 \mathrm{~b} \\
\text { 11-OH-THC: } 2.5 \mathrm{~b} \\
\text { THC-COOH: } 2.5 \mathrm{~b}\end{array}$ & \\
\hline & & & Oral & $\begin{array}{c}\text { Capsules } \\
\left.\text { (Marinol }^{\mathbb{B}}\right)\end{array}$ & $20 \mathrm{mg}$ THC & $\begin{array}{c}\text { THC: } 2.8 \text { (nd-5.6) b } \\
\text { 11-OH-THC: } 3.9 \text { (1.4-8.5) b } \\
\text { THC-COOH: } 27.8(5.4-55.4) \mathrm{b}\end{array}$ & $\begin{array}{c}\text { THC: } 1 \mathrm{~b} \\
\text { 11-OH-THC: } 4 \mathrm{~b} \\
\text { THC-COOH: } 5.5 \mathrm{~b}\end{array}$ & \\
\hline & & & Oral & $\begin{array}{l}\text { Decoction } \\
\text { (placebo) }\end{array}$ & $0.8 \mathrm{mg}$ THC & & & \\
\hline & & & Oral & $\begin{array}{l}\text { Capsules } \\
\text { (placebo) }\end{array}$ & & & & \\
\hline \multirow{5}{*}{$\begin{array}{l}\text { Goodwin et al., } \\
2006[43]\end{array}$} & \multirow{5}{*}{$\begin{array}{l}\mathrm{R}, \mathrm{DB}, \mathrm{P}, \mathrm{DD}, \\
\mathrm{MD}(5 \text { days })\end{array}$} & \multirow{5}{*}{$\begin{array}{c}6 \\
\begin{array}{l}\text { Previous experience of } \\
\text { cannabis use }\end{array}\end{array}$} & Oral & Hemp oil & $\begin{array}{c}0.39 \mathrm{mg} \text { THC/day } \\
\text { (tablespoon) }\end{array}$ & $\begin{array}{c}\text { THC: } 0 \mathrm{f}(0.0-0.0) \\
\text { 11-OH-THC: } 0 \mathrm{f}(0.0-0.0) \\
\text { THC-COOH: } 1.1 \mathrm{f}(0.0-3.1)\end{array}$ & $\begin{array}{c}\text { THC: } 0.0 \mathrm{f}(0.0-0.0) \\
\text { 11-OH-THC: } 0.0 \mathrm{f}(0.0-0.0) \\
\text { THC-COOH: } 49.7 \mathrm{f}(4.5-121)\end{array}$ & \multirow{5}{*}{$\begin{array}{c}\text { Subjective effects } \\
\text { Mild prototypical effects of THC. } \\
\text { Vital effects } \\
\text { No difference in } \mathrm{BP} \text {, HR, and respiratory rate }\end{array}$} \\
\hline & & & Oral & $\begin{array}{l}\text { Capsules (hemp } \\
\text { oil) }\end{array}$ & $0.47 \mathrm{mg} \mathrm{THC} /$ day & $\begin{array}{c}\text { THC: } 0.0 \mathrm{f}(0.0-0.0) \\
\text { 11-OH-THC: } 0.0 \mathrm{f}(0.0-0.0) \\
\text { THC-COOH: } 1.4 \mathrm{f}(0.0-2.6)\end{array}$ & $\begin{array}{c}\text { THC: } 0.0 \mathrm{f}(0.0-0.0) \\
\text { 11-OH-THC: } 0.0 \mathrm{f}(0.0-0.0) \\
\text { THC-COOH: } 65.3 \mathrm{f}(11.0-107)\end{array}$ & \\
\hline & & & Oral & $\begin{array}{l}\text { Capsules } \\
\text { (dronabinol) }\end{array}$ & 7.5 mg THC/day & $\begin{array}{c}\text { THC: } 1.5 \mathrm{f}(0.6-3.8) \\
\text { 11-OH-THC: } 1.6 \mathrm{f}(0.0-2.6) \\
\text { THC-COOH: } 19.8 \mathrm{f}(10.6-43.0)\end{array}$ & $\begin{array}{c}\text { THC: } 57.6 \mathrm{f}(6.5-107) \\
\text { 11-OH-THC: } 85.9 \mathrm{f}(1.5-107) \\
\text { THC-COOH: } 107 \mathrm{f}(107-107)\end{array}$ & \\
\hline & & & Oral & Hemp oil & $14,8 \mathrm{mg}$ THC/day & $\begin{array}{c}\text { THC: } 2.1 \mathrm{f} \text { (0.7-6.1) } \\
\text { 11-OH-THC: } 1.7 \mathrm{f}(0.0-5.6) \\
\text { THC-COOH: } 12.7 \mathrm{f}(11.0-15.2)\end{array}$ & $\begin{array}{c}\text { THC: } 56.5 \mathrm{f}(9-107) \\
\text { 11-OH-THC: } 28.6 \mathrm{f}(6.5-107) \\
\text { THC-COOH: } 91.5 \mathrm{f}(11.5-121)\end{array}$ & \\
\hline & & & Oral & Placebo & & & & \\
\hline
\end{tabular}


Table 2. Cont.

\begin{tabular}{|c|c|c|c|c|c|c|c|c|}
\hline References & Study Design & Participants & $\begin{array}{c}\text { Route of } \\
\text { Administration }\end{array}$ & Formulation & Dose & $\begin{array}{c}\text { Cmax (ng/mL) } \\
\text { Mean } \pm \text { SD, Median (Range) }\end{array}$ & $\operatorname{Tmax}(\mathrm{h})$ & Pharmacological Effects \\
\hline \multirow{6}{*}{ Pellesi et al., 2018 [52] } & \multirow{6}{*}{$\mathrm{OL}, \mathrm{C}$} & \multirow{6}{*}{$\begin{array}{l}6 \mathrm{M}, 7 \mathrm{~F} \\
\text { Patients with medication } \\
\text { overuse headaches }\end{array}$} & & & $1.85 \pm 1.6 \mathrm{mg} \mathrm{THC}$ & THC: $1.38 \pm 0.75$ & THC: $1.28 \pm 0.51$ & \multirow{6}{*}{$\begin{array}{l}\text { Subjective effects } \\
\text { Intensity of subjective effects was similar in } \\
\text { both formulations. Increased drowsiness after } \\
\text { cannabis oil administration. } \\
\text { Vital effects } \\
\text { No changes in BP and HR. }\end{array}$} \\
\hline & & & Oral & Decoction & $\begin{array}{c}2.22 \pm 0.66 \mathrm{mg} \\
\text { THCA-A }\end{array}$ & THCA: $48.92 \pm 26.34$ & THCA: $1.22 \pm 0.26$ & \\
\hline & & & & & $1.93 \pm 1.17 \mathrm{mg}$ CBD & CBD: $4.39 \pm 3.01$ & CBD: $0.56 \pm 0.17$ & \\
\hline & & & & & & CBDA: $74.61 \pm 25.15$ & CBDA: $0.83 \pm 0.35$ & \\
\hline & & & Oral & Oil & $\begin{array}{c}2.2 \mathrm{mg} \mathrm{THC} \\
2.3 \mathrm{mg} \mathrm{THCA}-\mathrm{A} \\
2.4 \mathrm{mg} \text { CBD }\end{array}$ & $\begin{array}{c}\text { THC: } 3.29 \pm 1.39 \\
\text { THCA: } 65.3 \pm \pm 20.40 \\
\text { CBD: } 3.14 \pm 2.58\end{array}$ & $\begin{array}{c}\text { THC: } 1.28 \pm 0.36 \\
\text { THCA: } 1.33 \pm 0.35 \\
\text { CBD: } 1 \pm 0.25\end{array}$ & \\
\hline & & & & & $4.4 \mathrm{mg}$ CBDA & CBDA: $55.03 \pm 29.45$ & CBDA: $1.06 \pm 0.3$ & \\
\hline \multirow{22}{*}{ Pichini et al., 2020 [53] } & \multirow{22}{*}{$\begin{array}{l}\text { NR, OL, NP } \\
\text { Pilot }\end{array}$} & \multirow{22}{*}{$1 \mathrm{M}$} & & & & Blood & Blood & \multirow{22}{*}{ Effects were not reported. } \\
\hline & & & & & & THC: 1.0 & THC: 2.0 & \\
\hline & & & & & & $\begin{array}{l}\text { THCA-A: } 72.4 \\
\text { CBD: } 1.5\end{array}$ & THCA-A: 2.0 & \\
\hline & & & & & & $\begin{array}{l}\text { CBDD: } 1.5 \\
\text { CBDA: } 94.3\end{array}$ & $\begin{array}{l}\text { CBD: } 3.0 \\
\text { CBDA: } 0.5\end{array}$ & \\
\hline & & & & & $\begin{array}{l}0.36 \mathrm{mg} \text { THC } \\
1.6 \mathrm{mg} \text { THCA-A }\end{array}$ & 11-OH-THC: 1.2 & 11-OH-THC: 2.0 & \\
\hline & & & Oral & Decoction & $0.42 \mathrm{mg}$ CBD & THC-COOH: 17.1 & THC-СOOH: 3.0 & \\
\hline & & & & & $4 \mathrm{mg}$ CBDA & THC-COOH-GLUC: 40.2 & THC-COOH-GLUC: 4.0 & \\
\hline & & & & & & $\begin{array}{l}\text { Oral fluid } \\
\text { THC. } 02\end{array}$ & $\begin{array}{l}\text { Oral fluid } \\
\text { THC. } 05\end{array}$ & \\
\hline & & & & & & THCA-A: 5.1 & THCA-A: 0.5 & \\
\hline & & & & & & CBD: 0.8 & CBD: 0.5 & \\
\hline & & & & & & CBDA: 145.2 & CBDA: 0.5 & \\
\hline & & & \multirow{11}{*}{ Oral } & \multirow{11}{*}{ Oil } & \multirow{11}{*}{$\begin{array}{c}0.95 \mathrm{mg} \mathrm{THC} \\
1.5 \mathrm{mg} \text { THCA-A } \\
0.86 \mathrm{mg} \text { CBD } \\
2.8 \mathrm{mg} \text { CBDA }\end{array}$} & Blood & Blood & \\
\hline & & & & & & THC: 0.5 & THC: 2.0 & \\
\hline & & & & & & $\begin{array}{l}\text { THCA: } 40.3 \\
\text { CBD }: 0.3\end{array}$ & THCA-A: 2.0 & \\
\hline & & & & & & CBDA: 32.4 & $\begin{array}{l}\text { CBD: } 2.0 \\
\text { CBDA: } 15\end{array}$ & \\
\hline & & & & & & 11-OH-THC: 0.7 & 11-OH-THC: 2.0 & \\
\hline & & & & & & THC-COOH: 4.3 & THC-СOOH: 2.0 & \\
\hline & & & & & & THC-COOH-GLUC: 7.7 & THC-COOH-GLUC: 3.0 & \\
\hline & & & & & & Oral fluid & Oral fluid & \\
\hline & & & & & & $\begin{array}{l}\text { THC: } 0.2 \\
\text { THCA: } 1.0\end{array}$ & $\begin{array}{c}\text { THC: } 2 \\
\text { THCA-A: } 2\end{array}$ & \\
\hline & & & & & & $\begin{array}{l}\text { THCA: } 1.0 \\
\text { CBD: } 0.6\end{array}$ & CBD: 2 & \\
\hline & & & & & & CBDA: 14.3 & CBDA: 1 & \\
\hline
\end{tabular}

For abbreviations see Table 1. 
Table 3. Summary of studies included in this systematic review reporting the pharmacokinetic parameters of THC following tablet administration.

\begin{tabular}{|c|c|c|c|c|c|c|c|c|}
\hline References & Study Design & Participants & $\begin{array}{c}\text { Route of } \\
\text { Administration }\end{array}$ & Formulation & Dose & $\begin{array}{c}\text { Cmax (ng/mL) } \\
\text { Mean } \pm \text { SD, Median (Range) }\end{array}$ & $\operatorname{Tmax}(\mathrm{h})$ & Pharmacological Effects \\
\hline \multirow{3}{*}{$\begin{array}{l}\text { Timpone et al., } \\
\quad 1997 \text { [54] }\end{array}$} & \multirow{3}{*}{$\mathrm{R}, \mathrm{OL}$} & \multirow{3}{*}{$\begin{array}{c}7 \mathrm{M} / \mathrm{F} \\
4 \mathrm{M} / \mathrm{F} \\
9 \mathrm{M} / \mathrm{F} \\
\text { Patients with HIV wasting } \\
\text { syndrome }\end{array}$} & Oral & $\begin{array}{c}\text { Tablets } \\
\left(\text { Marinol }^{\circledR}\right)\end{array}$ & $2.5 \mathrm{mg}$ THC & Data from all 20 patients & Data from all 20 patients & \multirow{3}{*}{$\begin{array}{c}\text { Subjective effects } \\
\text { Increase in VAS for hunger. } \\
\text { No differences in VAS for mood and nausea. }\end{array}$} \\
\hline & & & Oral & $\begin{array}{c}\text { Tablets } \\
\left(\text { Marinol }^{\mathbb{B}}\right)\end{array}$ & $\begin{array}{c}2.5 \mathrm{mg} \text { THC } \\
750 \mathrm{mg} \text { megestrol }\end{array}$ & THC: 2.01 c (0.58-12.48) & THC: $2.07 b(0.66-8.26)$ & \\
\hline & & & Oral & $\begin{array}{c}\text { Tablets } \\
\left(\text { Marinol }^{\mathbb{Q}}\right)\end{array}$ & $\begin{array}{c}2.5 \mathrm{mg} \text { THC } \\
250 \mathrm{mg} \text { megestrol }\end{array}$ & $11-\mathrm{OH}-\mathrm{THC}: 4.61$ c (0.52-37.5) & $11-\mathrm{OH}-\mathrm{THC}: 2.07 b(0.49-8.00)$ & \\
\hline \multirow{5}{*}{$\begin{array}{l}\text { Klumpers et al., } \\
2011 \text { [55] }\end{array}$} & \multirow{5}{*}{$\mathrm{R}, \mathrm{DB}, \mathrm{DD}, \mathrm{P}, \mathrm{C}$} & \multirow{5}{*}{$\begin{array}{c}4 \mathrm{M}, 5 \mathrm{~F} \\
\text { (in panel } 1,13 \text { subjects) } \\
\text { Previous experience of } \\
\text { cannabis use (maximum } 1 \text { use } \\
\text { per week) }\end{array}$} & Sublingual & $\begin{array}{c}\text { Tablets } \\
\left(\text { Namisol }^{\circledR}\right)\end{array}$ & $5.0 \mathrm{mg}$ THC & $2.30 \pm 1.01^{\mathrm{e}}$ & $1.24 \pm 0.65^{\mathrm{e}}$ & \multirow{5}{*}{$\begin{array}{l}\text { Subjective effects } \\
\text { Highest oral doses increased body sway and VAS for } \\
\text { calmness, external perception, and feeling high and } \\
\text { decreased VAS for alertness. } \\
\text { Vital effects } \\
\text { No significant differences in PD parameters between oral } \\
\text { and sublingual administration. } \\
\text { Significant increase in HR. }\end{array}$} \\
\hline & & & Oral & $\begin{array}{c}\text { Tablets } \\
\left(\text { Namisol }^{\mathbb{R}}\right)\end{array}$ & $5.0 \mathrm{mg}$ THC & $2.92 \pm 1.49^{\mathrm{e}}$ & $0.93 \pm 0.68^{\mathrm{e}}$ & \\
\hline & & & Oral & $\begin{array}{c}\text { Tablets } \\
\left(\text { Namisol }^{\circledR}\right)\end{array}$ & $6.5 \mathrm{mg}$ THC & $4.43 \pm 1.86^{\mathrm{e}}$ & $0.66 \pm 0.13^{e}$ & \\
\hline & & & Oral & $\begin{array}{c}\text { Tablets } \\
\left(\text { Namisol }^{\mathbb{Q}}\right)\end{array}$ & $8.0 \mathrm{mg}$ THC & $4.69 \pm 2.91^{\mathrm{e}}$ & $0.73 \pm 0.19^{\mathrm{e}}$ & \\
\hline & & & Oral & $\begin{array}{c}\text { Tablets } \\
\text { (placebo) }\end{array}$ & & & & \\
\hline \multirow{4}{*}{$\begin{array}{l}\text { Ahmed et al., } \\
2014[56]\end{array}$} & \multirow{4}{*}{$\mathrm{R}, \mathrm{DB}, \mathrm{P}, \mathrm{DD}, \mathrm{C}$} & \multirow{4}{*}{$6 \mathrm{M}, 5 \mathrm{~F}$} & Oral & $\begin{array}{c}\text { Tablets } \\
\left(\text { Namisol }^{\circledR}\right)\end{array}$ & $3 \mathrm{mg} \mathrm{THC}$ & $1.42(0.53-3.48)$ & $0.92(0.67-0.92)$ & \multirow{4}{*}{$\begin{array}{c}\text { Subjective effects } \\
\text { No subjective effects (exc. } 4 \text { subjects "felt high") } \\
\text { Vital effects } \\
\text { Mild PD effects. } \\
\text { No changes in SBP, DBP, and HR. } \\
\text { Psychomotor performance } \\
\text { No changes in psychomotor performance. }\end{array}$} \\
\hline & & & Oral & $\begin{array}{c}\text { Tablets } \\
\left(\text { Namisol }^{\circledR}\right) \\
\end{array}$ & $5 \mathrm{mg}$ THC & $3.15(1.54-6.95)$ & $0.92(0.67-0.92)$ & \\
\hline & & & Oral & $\begin{array}{c}\text { Tablets } \\
\left(\text { (Namisol }^{\circledR}\right)\end{array}$ & $6.5 \mathrm{mg}$ THC & $4.57(2.11-8.65)$ & $0.67(0.67-0.92)$ & \\
\hline & & & Oral & $\begin{array}{c}\text { Tablets } \\
\text { (placebo) }\end{array}$ & & & & \\
\hline \multirow{2}{*}{$\begin{array}{l}\text { De Vries et al., } \\
2016[57]\end{array}$} & \multirow[b]{2}{*}{$\mathrm{R}, \mathrm{DB}, \mathrm{P}, \mathrm{C}$} & \multirow{2}{*}{$\begin{array}{l}15 \mathrm{M}, 9 \mathrm{~F} \\
\text { Patients diagnosed with } \\
\text { chronic pancreatitis } \\
\text { No cannabis use in previous } \\
\text { year }\end{array}$} & Oral & $\begin{array}{c}\text { Tablets } \\
\text { (Namisol }{ }^{\mathbb{B}} \text { ) }\end{array}$ & $8 \mathrm{mg} \mathrm{THC}$ & $\begin{array}{c}\text { THC: } 4.01 \pm 3.39 \\
11-\mathrm{OH}-\mathrm{THC}: 4.38 \pm 1.50\end{array}$ & $\begin{array}{l}2.05+1.47 \\
2.26 \pm 1.29\end{array}$ & \multirow{2}{*}{$\begin{array}{l}\text { Subjective effects No differences in subjective effects } \\
\text { (alertness, mood, calmness, or balance) between treatments. } \\
\text { Anxiousness, somnolence, dry mouth, dizziness, and } \\
\text { euphoric mood after THC administration. Vital effects No } \\
\text { changes in SBP and DBP. THC induced an increase in HR } \\
\text { compared to diazepam. }\end{array}$} \\
\hline & & & Oral & $\begin{array}{l}\text { Tablet (active } \\
\text { placebo) }\end{array}$ & $\begin{array}{l}5 \mathrm{mg} \text { diazepam to } \\
\text { non-opioid group } / 10 \\
\text { mg diazepam to } \\
\text { opioid group }\end{array}$ & & & \\
\hline
\end{tabular}


Table 4. Summary of studies included in this systematic review reporting the pharmacokinetic parameters for THC following baked goods' administration.

\begin{tabular}{|c|c|c|c|c|c|c|c|c|}
\hline References & Study Design & Participants & $\begin{array}{c}\text { Route of } \\
\text { Administration }\end{array}$ & Formulation & Dose & $\begin{array}{c}\text { Cmax }(\mathrm{ng} / \mathrm{mL}) \\
\text { Mean } \pm \text { SD, Median (Range) }\end{array}$ & $\operatorname{Tmax}(\mathbf{h})$ & Pharmacological Effects \\
\hline \multirow{3}{*}{$\begin{array}{l}\text { Ohlsson et al., } \\
\quad 1980[58]\end{array}$} & \multirow{3}{*}{$\mathrm{R}, \mathrm{OL}, \mathrm{NP}, \mathrm{C}$} & \multirow{3}{*}{$\begin{array}{c}11 \mathrm{M} \\
\text { Previous experience of } \\
\text { cannabis use (from infrequent } \\
\text { to frequent use) }\end{array}$} & Smoked & Cigarette & $\begin{array}{c}19 \mathrm{mg} \mathrm{THC} \mathrm{(ad} \\
\text { libitum) (mean = } 13.0 \\
\mathrm{mg})\end{array}$ & $77,33-118^{\text {a }}$ & & \multirow{3}{*}{$\begin{array}{l}\text { Subjective effects } \\
\text { Increase in high effect. } \\
\text { Vital effects } \\
\text { Increase in HR. } \\
\text { Conjunctival reddening }\end{array}$} \\
\hline & & & Oral & Chocolate cookie & $20 \mathrm{mg}$ THC & $4.4-11^{\mathrm{a}}$ & $1-1.5$ & \\
\hline & & & Intravenous & $\begin{array}{l}\text { Normal saline ethanolic } \\
\text { solution }\end{array}$ & $5 \mathrm{mg} \mathrm{THC}$ & $219,161-316^{\text {a }}$ & & \\
\hline \multirow{10}{*}{$\begin{array}{l}\text { Watchel et al., } \\
2002 \text { [59] }\end{array}$} & \multirow{10}{*}{$\mathrm{DB}, \mathrm{P}, \mathrm{C}$} & \multirow{10}{*}{$\begin{array}{c}7 \mathrm{M}, 5 \mathrm{~F} \\
7 \mathrm{M}, 6 \mathrm{~F} \\
\begin{array}{c}\text { Previous experience of } \\
\text { cannabis use }\end{array}\end{array}$} & Oral & Cannabis (plant) brownie & $8.4 \mathrm{mg} \mathrm{THC}$ & $\approx 4.1^{\mathrm{b}, \mathrm{d}}$ & $3^{b, d}$ & \multirow{10}{*}{$\begin{array}{l}\text { Subjective effects } \\
\text { Both drugs increased VAS } \\
\text { sedation and ARCI PCAG scale } \\
\text { scores, and decreased the ARCI } \\
\text { BG scale scores at higher doses } \\
\text { Cannabis in high doses } \\
\text { increased VAS for sedation, } \\
\text { drowsiness, and tiredness. } \\
\text { THC in high doses increased } \\
\text { ARCI A scale scores, MBG } \\
\text { (euphoria), and LSD } \\
\text { (dysphoria). Vital effects No } \\
\text { effects on physiological or } \\
\text { behavioral measures. }\end{array}$} \\
\hline & & & Oral & Cannabis (plant) brownie & $16.9 \mathrm{mg} \mathrm{THC}$ & $\approx 6.8^{\mathrm{b}, \mathrm{d}}$ & $2.5 \mathrm{~b}, \mathrm{~d}$ & \\
\hline & & & Oral & THC (synthetic) brownie & $8.4 \mathrm{mg}$ THC & $\approx 4.8^{\mathrm{b}, \mathrm{d}}$ & $2.5^{\mathrm{b}, \mathrm{d}}$ & \\
\hline & & & Oral & THC (synthetic) brownie & $16.9 \mathrm{mg}$ THC & $\approx 9^{\mathrm{b}, \mathrm{d}}$ & $2.5^{\mathrm{b}, \mathrm{d}}$ & \\
\hline & & & Smoked & Cannabis (plant) cigarette & $8.4 \mathrm{mg} \mathrm{THC}$ & $\approx 36^{\mathrm{b}, \mathrm{d}}$ & $0.08^{b, d}$ & \\
\hline & & & Smoked & Cannabis (plant) cigarette & $16.9 \mathrm{mg} \mathrm{THC}$ & $\approx 60^{\mathrm{b}, \mathrm{d}}$ & $0.08^{b, d}$ & \\
\hline & & & Smoked & THC (synthetic) cigarette & $8.4 \mathrm{mg} \mathrm{THC}$ & $\approx 31^{\mathrm{b}, \mathrm{d}}$ & $0.08^{\mathrm{b}, \mathrm{d}}$ & \\
\hline & & & Smoked & THC (synthetic) cigarette & $16.9 \mathrm{mg}$ THC & $\approx 56^{\mathrm{b}, \mathrm{d}}$ & $0.08^{b, d}$ & \\
\hline & & & Oral & Brownie (placebo) & & & & \\
\hline & & & Smoked & Cigarette (placebo) & & & & \\
\hline \multirow{19}{*}{$\begin{array}{l}\text { Newmeyer } \\
\text { et al., 2016 [60] }\end{array}$} & \multirow{19}{*}{$\mathrm{R}, \mathrm{DB}, \mathrm{P}, \mathrm{DD}, \mathrm{C}$} & \multirow{19}{*}{$\begin{array}{l}9 \mathrm{M}, 2 \mathrm{~F} \text { frequent smokers } \\
6 \mathrm{M}, 3 \mathrm{~F} \text { occasional smokers }\end{array}$} & \multirow{11}{*}{ Oral } & \multirow{11}{*}{ Brownie } & \multirow{11}{*}{$\begin{array}{l}50.6 \mathrm{mg} \mathrm{THC}(\mathrm{ad} \\
\text { libitum) } \\
1.5 \mathrm{mg} \mathrm{CBD} \\
3.3 \mathrm{mg} \mathrm{CBN}\end{array}$} & \multirow{11}{*}{ 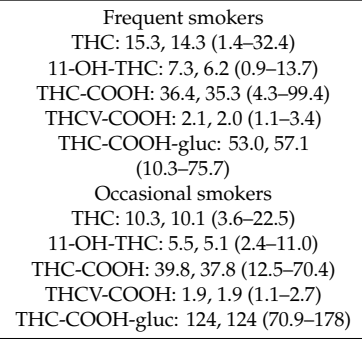 } & Frequent smokers & \multirow{19}{*}{ Effects were not described. } \\
\hline & & & & & & & THC: $2.5,2.5(1.5-3.5)$ & \\
\hline & & & & & & & 11-OH-THC: $2.3,2.5$ (1.5-3.5) & \\
\hline & & & & & & & $\begin{array}{l}\text { 1HC-COOH: } 2.7,2.5(2.53-3.5) \\
\text { THCV-COOH: } 3.0,3.0(2.5-3.5)\end{array}$ & \\
\hline & & & & & & & THCOOH-gluc: $3.4,3.5$ (1.5-5.0) & \\
\hline & & & & & & & Occasional smokers & \\
\hline & & & & & & & THC: $2.3,2.5$ (1.5-3.5) & \\
\hline & & & & & & & 11-OH-THC: $2.4,2.5$ (1.5-3.5) & \\
\hline & & & & & & & THC-COOH: $2.9,3.5(1.5-3.5)$ & \\
\hline & & & & & & & THCV-COOH: $2.6,2.5$ (1.5-3.5) & \\
\hline & & & & & & & THC-COOH-gluc: 4.7, 5.0 (3.5-5.0) & \\
\hline & & & \multirow{8}{*}{ Smoked } & \multirow{8}{*}{ Cigarette } & & $\begin{array}{c}\text { Frequent smokers } \\
\mathrm{THC}: 151,114(51.6-467)\end{array}$ & $\begin{array}{l}\text { Frequent smokers } \\
\end{array}$ & \\
\hline & & & & & & 11-OH-THC: $9.0,6.5$ (1.9-30.2) & 11-OH-THC: $0.21,0.20(0.10-0.50)$ & \\
\hline & & & & & & THC-COOH: 23.5, 20.0 (5.7-64.9) & THC-COOH: $0.28,0.25(0.00-0.50)$ & \\
\hline & & & & & $\begin{array}{l}50.6 \mathrm{mg} \mathrm{THC}(\mathrm{ad} \\
\text { libitum) }\end{array}$ & 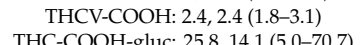 & THCV-COOH: $0.22,0.23(0.17-0.25)$ & \\
\hline & & & & & $1.5 \mathrm{mg}$ CBD & $\begin{array}{l}\text { THC-COOH-gluc: 25.8, 14.1 (5.0-70.7) } \\
\text { Occasional smokers }\end{array}$ & & \\
\hline & & & & & $3.3 \mathrm{mg}$ CBN & THC: $51.6,44.4(1.3-174)$ & THC: $0.11,0.10(0.07-0.17)$ & \\
\hline & & & & & & $\begin{array}{l}\text { 11-OH-THC: } 2.8,1.9(0.5-8.7) \\
\text { THC-COOH: } 84.74(0 .-175)\end{array}$ & $\begin{array}{l}\text { 11-OH-THC: } 0.22,0.19(0.10-0.50) \\
\text { THC-COOH. } 031.025(0.10-5050\end{array}$ & \\
\hline & & & & & & $\begin{array}{l}\text { THC-COOH: 8.4, 7.4 (0.7-17.5) } \\
\text { THCV-COOH: - }\end{array}$ & $\begin{array}{c}\text { THC-COOH: } 0.31,0.25(0.10-0.50) \\
\text { THCVCOOH: - }\end{array}$ & \\
\hline
\end{tabular}


Table 4. Cont.

\begin{tabular}{|c|c|c|c|c|c|c|c|c|}
\hline References & Study Design & Participants & $\begin{array}{c}\text { Route of } \\
\text { Administration }\end{array}$ & Formulation & Dose & $\begin{array}{c}\text { Cmax (ng/mL) } \\
\text { Mean } \pm \text { SD, Median (Range) }\end{array}$ & $\operatorname{Tmax}(\mathrm{h})$ & Pharmacological Effects \\
\hline & & & Inhaled & Vaporizer Volcano & $\begin{array}{c}50.6 \mathrm{mg} \mathrm{THC}(\mathrm{ad} \\
\text { libitum) } \\
1.5 \mathrm{mg} \mathrm{CBD} \\
3.3 \mathrm{mg} \mathrm{CBN}\end{array}$ & 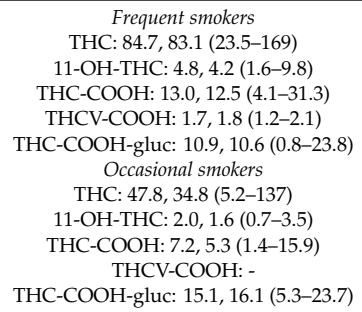 & $\begin{array}{c}\text { Frequent smokers } \\
\text { THC: } 0.09,0.10(0.03-0.17) \\
\text { 11-OH-THC: } 0.19,0.17(0.10-0.50) \\
\text { THC-COOOH: } 0.25,0.25(0.13-0.50) \\
\text { THCV-COOH: } 0.52,0.21(0.17-1.5) \\
\text { THC-COOH-gluc: } 1.8,1.5(0.03-3.5) \\
\text { Occasional smokers } \\
\text { THC: } 0.11,0.10(0.03-0.17) \\
\text { 11-OH-THC: } 0.15,0.15(0.10-0.20) \\
\text { THC-COOH: } 0.33,0.25(0.20-0.50) \\
\text { THCV-COOH:- } \\
\text { THC-COOH-gluc: } 1.9,2.5(0.5-2.5)\end{array}$ & \\
\hline & & & Oral & Brownie (placebo) & & & & \\
\hline & & & Smoked & Cigarette (placebo) & & & & \\
\hline & & & Inhaled & Vaporizer (placebo) & & & & \\
\hline $\begin{array}{l}\text { Newmeyer } \\
\text { et al., 2017 [61] }\end{array}$ & $\begin{array}{c}\text { Optional dosing } \\
\text { session under the } \\
\text { same clinical protocol } \\
\text { followed in } \\
\text { Newmeyer et al., } 2016\end{array}$ & $\begin{array}{c}9 \mathrm{M} \text { frequent smokers } \\
5 \mathrm{M}, 2 \mathrm{~F} \text { occasional smokers }\end{array}$ & Oral & Brownie & $\begin{array}{l}50.6 \mathrm{mg} \text { THC } \\
1.5 \mathrm{mg} \text { CBD } \\
3.3 \mathrm{mg} \text { CBN } \\
\text { (ad libitum) }\end{array}$ & 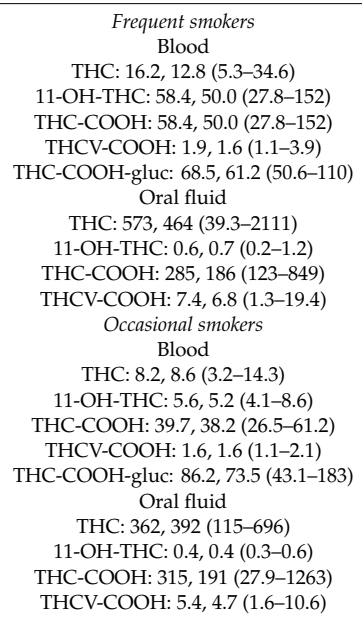 & 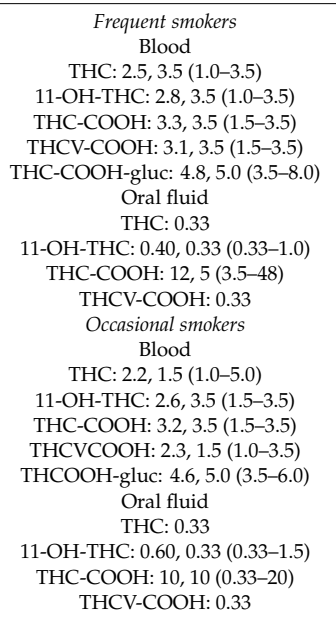 & Effects were not assessed. \\
\hline
\end{tabular}


Table 4. Cont.

\begin{tabular}{|c|c|c|c|c|c|c|c|c|}
\hline References & Study Design & Participants & $\begin{array}{c}\text { Route of } \\
\text { Administration }\end{array}$ & Formulation & Dose & $\begin{array}{c}\text { Cmax }(\mathrm{ng} / \mathrm{mL}) \\
\text { Mean } \pm \text { SD, Median (Range) }\end{array}$ & $\operatorname{Tmax}(\mathrm{h})$ & Pharmacological Effects \\
\hline \multirow{3}{*}{$\begin{array}{l}\text { Vandrey et al., } \\
2017[62]\end{array}$} & \multirow{3}{*}{$\mathrm{R}, \mathrm{DB}, \mathrm{NP}$} & \multirow{3}{*}{$\begin{array}{c}9 \mathrm{M}, 9 \mathrm{~F} \\
\text { Previous experience of } \\
\text { cannabis use but not exposed } \\
\text { within the previous } 3 \text { months }\end{array}$} & Oral & Brownie & $10 \mathrm{mg}$ THC & $\begin{array}{c}\text { Blood } \\
\text { THC: } 1.0(0-3) \\
\text { 11-OH-THC: } 1.0(0-2) \\
\text { THC-COOH: } 7.2(5-14) \\
\text { Oral fluid } \\
\text { THC: } 191.5(47-412) \\
\text { THC-COOH: } 0.051 \text { (0-0.231) }\end{array}$ & $\begin{array}{c}\text { Blood } \\
\text { THC: } 0.9(0-2) \\
\text { 11-OH-THC } 1.3(0-3) \\
\text { THC-COOH: } 3.2(2-4) \\
\text { Oral fluid } \\
\text { THC: } 0.2(0.2-0.5) \\
\text { THC-COOH: } 1.0(0-3)\end{array}$ & \multirow{3}{*}{$\begin{array}{c}\text { Subjective effects } \\
\text { Significant subjective and } \\
\text { cognitive drug effects at the } 25 \\
\text { and } 0 \mathrm{mg} \text { doses. } \\
\text { Vital effects } \\
\text { Significant PD effects. } \\
\text { Psychomotor performance } \\
\text { Significant effects on } \\
\text { psychomotor performance at } \\
\text { the } 25 \text { and } 50 \mathrm{mg} \text { doses. }\end{array}$} \\
\hline & & & Oral & Brownie & $25 \mathrm{mg}$ THC & $\begin{array}{c}\text { Blood } \\
\text { THC: } 3.5(3.0-4) \\
\text { 11-OH-THC: } 3.3(2-5) \\
\text { THC-COOH: } 21.3(12-39) \\
\text { Oral fluid } \\
\text { THC: } 477.5(70-1128) \\
\text { THC-COOH: } 0.140(0.023-0.251)\end{array}$ & $\begin{array}{c}\text { Blood } \\
\text { THC: } 2.6(1.0-4) \\
\text { 11-OH-THC: } 3.0(1.5-4) \\
\text { THC-COOH: } 3.3(1.5-6) \\
\text { Oral fluid } \\
\text { THC: } 0.2(0.2-0.5) \\
\text { THC-COOH: } 9.8(3-30) \\
\end{array}$ & \\
\hline & & & Oral & Brownie & $50 \mathrm{mg}$ THC & $\begin{array}{c}\text { Blood } \\
\text { THC: } 3.3(1.0-5) \\
\text { 11-OH-TH: } 3.2(2-4) \\
\text { THC-COOH: } 29.3(16-44) \\
\text { Oral fluid } \\
\text { THC: 597.5 (350-1010) } \\
\text { THC-COOH: } 0.314(0-0.822)\end{array}$ & $\begin{array}{c}\text { Blood } \\
\text { THC: } 2.3(1.0-6) \\
\text { 11-OH-THC } 1.8(1-3) \\
\text { THC-COOH: } 3.3(1.5-6) \\
\text { Oral fluid } \\
\text { THC: } 0.2(0.2-0.5) \\
\text { THC-COOH: } 17.4(0-54)\end{array}$ & \\
\hline
\end{tabular}

For abbreviations, see Table 1. 
Overall, these studies showed remarkable heterogeneity in their designs and the conditions under which they were conducted. Most studies included small sample sizes, and not all of them included subjects of both genders. There were also discrepancies in the cannabis experience levels of the participants and their health statuses, since patients with diverse pathologies (HIV, chronic pancreatitis, and medication overuse-related headaches) were targeted in several studies. Most of the studies involved THC administration alone, although THC was administered in combination with CBD in eight studies. Apart from other cannabinoids, THC was also administered combined with other drugs, such as megestrol acetate, naltrexone, and morphine.

Some studies included evaluations of physiological and/or subjective effects after administration of the different preparations. THC/cannabis was found to produce its prototypical effects, presenting mild changes in blood pressure or heart rate, increases in scores of high and positive effects, increased feelings of sedation/drowsiness, and mild impairment of psychomotor performance. See Tables 1-4 for detailed descriptions of these effects in different studies according to various formulations.

\subsection{Capsules}

Cannabis capsules usually contain cannabis or synthetic THC (dronabinol) in oil due to its higher bioavailability (see also the oil section). In our search, 14 studies investigated cannabis/THC/dronabinol administration dissolved in oil capsules, thus representing the most frequently studied dosage form among all oral formulations [37-50].

The dose of THC contained in the oil capsules in single-dose studies ranged from 5 to $90 \mathrm{mg}$. The Cmax in plasma ranged from 0.42 to $29.9 \mathrm{ng} / \mathrm{mL}$, and the Tmax ranged from 0.78 to $4 \mathrm{~h}$. PK differences were examined after administering the same cannabinoid doses (10.8 $\mathrm{mg}$ of THC and $10.0 \mathrm{mg}$ of CBD) using THC and CBD-piperine-pro-nanolipospheres (THC-CBD-PNL) capsules, which are an alternative to oil capsules, and an oromucosal spray (Sativex ${ }^{\circledR}$ ). THC-CBD-PNL produced a three-fold increase in Cmax compared to Sativex ${ }^{\circledR}$ (5.4 and $1.8 \mathrm{ng} / \mathrm{mL}$ of THC, respectively) and a faster absorption of cannabinoids (a Tmax of $1 \mathrm{~h}$ and $2 \mathrm{~h}$ for THC and of $1 \mathrm{~h}$ and $2 \mathrm{~h}$ for CBD, respectively) [51]. See Table 1 for the specific results.

The correlation between the administered dose and Cmax resulted in a Pearson's $r$ value of 0.9271 and a coefficient of determination $\left(R^{2}\right)$ of 0.8596 (Table 5, Figure 2). The THC Cmax increased proportionally by increasing the doses of THC.

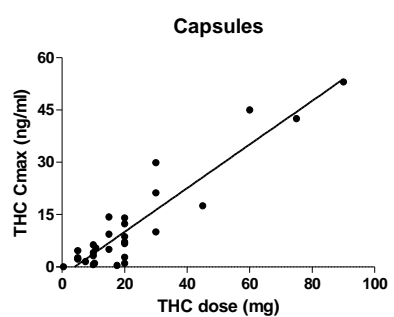

(a)

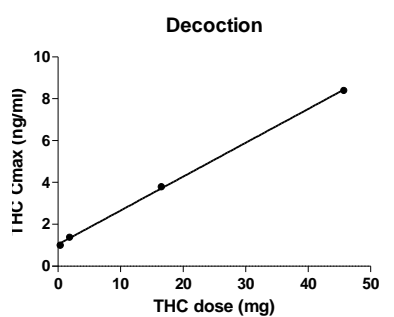

(b)

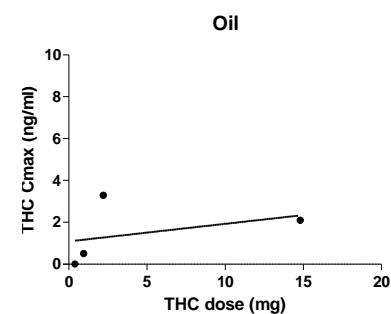

(c)

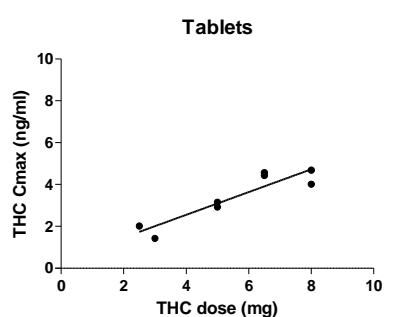

(d)

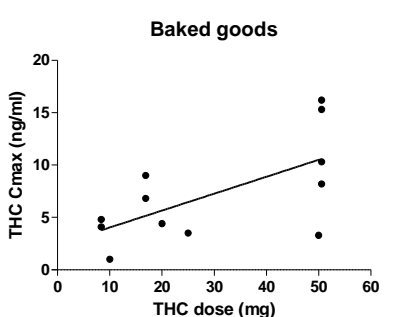

(e)

Figure 2. The correlation between the dose of administered THC and the maximum concentration (Cmax) of THC in plasma following administration of capsules (a), decoctions (b), oils (c), tablets (d), and baked goods (e). 
Table 5. Correlation between doses of THC and the maximum plasma concentration (Cmax) values in each formulation group.

\begin{tabular}{cccccc}
\hline & Capsules & Decoction & Oil & Tablets & Baked Goods \\
\hline Pearson's $r$ & 0.9271 & 0.9997 & 0.3806 & 0.9178 & 0.6365 \\
95\% confidence interval & 0.8492 to 0.9656 & 0.9851 to 1.000 & -0.9154 to 0.9824 & 0.6032 to 0.9853 & 0.09838 to 0.8866 \\
$p$ value (two-tailed) & $<0.0001$ & 0,0003 & 0.6194 & 0.0013 & 0.0261 \\
$\mathrm{R}^{2}$ & 0.8596 & 0.9994 & 0.1448 & 0.8423 & 0.4051 \\
\hline
\end{tabular}

\subsection{Oil}

In previous studies, oil extracts showed greater cannabinoid extraction efficiency than water [31]. In addition to a higher bioavailability, oil formulations are considered suitable solvents to compose a THC therapeutic preparation. In this section, we only considered the administration of oil-based cannabis (see above for capsules containing oil). Only three studies were found in which oil was directly ingested $[43,52,53]$. Among these three studies, only two reported single-dose administrations. In one of these studies, subjects received $2.2 \mathrm{mg}$ of THC and $2.3 \mathrm{mg}$ of $\Delta$-9-tetrahydrocannabinolic acid A (THCA-A), obtaining a Cmax of 3.29 and $65.36 \mathrm{ng} / \mathrm{mL}$, respectively, and a Tmax of 1.28 and $1.33 \mathrm{~h}$ in plasma [52]. The other study reported data on one healthy individual treated with a cannabis decoction and oil (pilot study). The subject received $0.45 \mathrm{~mL}$ of oil containing $0.95 \mathrm{mg}$ of THC, $1.5 \mathrm{mg}$ of THCA-A, $0.86 \mathrm{mg}$ of CBD, and $2.8 \mathrm{mg}$ of cannabidiolic acid (CBDA). The THC and THCA-A Cmax in serum following oil administration were 0.5 and $40.3 \mathrm{ng} / \mathrm{mL}$, respectively, with a Tmax of $2.0 \mathrm{~h}$ for both cannabinoids [53]. See Table 2 for the specific results.

In this formulation, among the three studies included, the administered dose of THC showed a weak and not significant correlation with the Cmax (Pearson's $r=0.3806, p$ value $=0.6194)$ (Table 5, Figure 2).

\subsection{Decoctions}

Decoctions are also called "tea" in several articles. Only three studies examining cannabinoid PK after cannabis decoction administration were retrieved, including one study with a milk decoction $[41,52,53]$. For the milk decoction, two doses were selected-a low THC dose of $16.5 \mathrm{mg}$ and a high dose of $45.7 \mathrm{mg}$, achieving a Cmax of 3.8 and $8.4 \mathrm{ng} / \mathrm{mL}$, respectively, and a Tmax of $1 \mathrm{~h}$ in plasma for both doses [41]. In another study, cannabis was boiled in water, obtaining a decoction composed of $1.85 \mathrm{mg}$ THC and $2.22 \mathrm{mg}$ of THCA-A. After consumption of this decoction, the THC reached a mean Cmax of $1.38 \mathrm{ng} / \mathrm{mL}$ with a Tmax of $1.28 \mathrm{~h}$, whereas THCA-A reached a mean Cmax of $48.92 \mathrm{ng} / \mathrm{mL}$ in plasma with a Tmax of $1.22 \mathrm{~h}$ [52]. In the pilot study mentioned in the Oil section, the subject received $100 \mathrm{~mL}$ of a cannabis decoction containing $0.36 \mathrm{mg}$ of THC, $1.6 \mathrm{mg}$ of THCA-A, $0.42 \mathrm{mg}$ of $\mathrm{CBD}$, and $4 \mathrm{mg}$ of CBDA. This oral dose resulted in a Cmax in the serum of $1.0 \mathrm{ng} / \mathrm{mL}$ of THC and $72.4 \mathrm{ng} / \mathrm{mL}$ of THCA-A, with a Tmax of $2.0 \mathrm{~h}$ [53]. See Table 2 for the specific results.

Despite the few studies found, cannabis decoctions showed a significant strong correlation between the dose of THC and peak plasma, with a Pearson's $r$ of 0.9997 and a correlation coefficient of $>0.99$ (Table 5, Figure 2).

\subsection{Tablets}

Like oral capsules, tablets are also a stable dosage form that is considered practical for patient use. Four studies were retrieved. Two of them focused on Namisol, a patented tablet formulation of pure THC under investigation [54-57]. The PK data for THC doses varied from 2.5 to $8 \mathrm{mg}$, producing a Cmax of $1.42-4.69 \mathrm{ng} / \mathrm{mL}$ and a Tmax of $0.66-2.07 \mathrm{~h}$ in plasma. See Table 3 for the specific results.

Tablet administration showed a strong correlation between the administered THC dose and the Cmax, with a Pearson's $r$ of 0.9178 and a correlation coefficient of 0.8423 (Table 5, Figure 2). 


\subsection{Baked Goods}

Five studies evaluated the THC PK after brownie or cookie consumption $[38,40,58,59,61]$. The THC doses in these edibles ranged from 8.4 to $50.6 \mathrm{mg}$, resulting in a Cmax of 1-16.2 ng/mL and a Tmax of $0.9-2.6 \mathrm{~h}$ in plasma. See Table 4 for specific results.

Brownies and cookies showed a weaker correlation between the dose of THC and the Cmax compared to other formulations, resulting in a Pearson's $r$ of 0.6365 and a correlation coefficient of 0.4051 (Table 5, Figure 2).

\section{Discussion}

The purpose of this present review was to provide a general overview of the available THC PK data after oral administration. In our literature review, we found that human PK studies studying the administration of THC in oral forms were scarce, despite their increasing popularity. Most of these studies focused on pharmaceutical forms, such as capsules and tablets. Despite being recommended formulations for the therapeutic use of cannabis in some countries, there were few data on the cannabinoid PK after the ingestion of cannabis oils or decoctions. The only complete, published study comparing these two formulations in patients with medication overuse-related headaches found high variability in the cannabinoid content of these formulations and in the THC recovery after administration. Each preparation showed differences in cannabinoid and metabolite absorption. For instance, cannabis decoctions offered a higher bioavailability of CBDA, while cannabis oil provided a higher bioavailability of THC and its metabolites, 11-hydroxy- $\Delta-9$-tetrahidrocannabinol (11-OH-THC) and 11-nor-9-carboxy- $\Delta$-9-tetrahidrocannabinol (THC-COOH) [52]. Contrary to these results, a published pilot study that also compared oil and decoction formulations obtained higher CBDA, THC, 11-OH-THC, and THC-COOH Cmax values after the administration of decoctions [53]. However, this pilot only presented data from one subject; results from a larger number of participants would likely strengthen comparisons between these two studies.

In our review, we found three studies that compared the oral administration of THC with that of smoking/inhalation, the most common route of administration. In Ohlsson et al. [58], subjects smoked $19.0 \mathrm{mg}$ of THC (ad libitum, with a mean of $13.0 \mathrm{mg}$ of THC) and took an oral dose of $20 \mathrm{mg}$ of THC via a chocolate cookie. Despite being administered in a similar dose, the Cmax obtained after smoking (33-118 $\mathrm{ng} / \mathrm{mL}$ ) was significantly higher than the oral administration $(4.4-11 \mathrm{ng} / \mathrm{mL})$. These results showed the low systemic bioavailability of oral THC, which is about a third of that from smoked THC. Similarly, in Watchel et al. [59], the same doses of synthetic or plant-derived THC were orally administered and smoked, but the Cmax obtained was six times higher after smoking than after oral administration. As expected, the oral form achieved a delayed Tmax since absorption is slower when cannabinoids are ingested. Newmeyer et al. [60] reported minimal differences between smoked and vaporized cannabis administration, observing similar delivery. However, the THC Cmax after oral administration (brownie) was significantly lower than that of other routes, in addition to having a delayed Tmax, which agrees with previous observations.

For capsules, a wider range of doses was evaluated compared to other forms. Lile et al. administered the highest dose of THC $(90 \mathrm{mg})$ out of all the studies and consequently reported the highest THC Cmax mean value obtained after administration (approx. $53 \mathrm{ng} / \mathrm{mL}$ ) [49].

Oral THC doses were moderately to highly correlated (Pearson's $r=0.6365-0.9997$ and $\mathrm{R}^{2}=0.4051-0.9994$ ), with peak plasma concentrations of THC found in capsules, decoctions, tablets, and baked goods formulations (Table 5, Figure 2). This correlation was stronger for capsules, decoctions, and oil compared to baked goods, which appeared to have higher variability in the peak plasma value obtained after certain THC doses. Interestingly, for oil, the correlation was not significant, thereby suggesting a more irregular absorption profile (Figure 2). Since THC is a highly lipophilic molecule, it is expected that its absorption will increase in oil-based formulations. More studies on cannabis oil PK could determine whether this lack of linear correlation, as proven in other formulations, persists due to a high variability in the THC absorption of cannabis oil. 
The management of dosing is critical for the treatment of patients, as there is a balance between the desired medical effects of THC and the prevention of adverse effects. Analyzing and understanding the PK of oral THC preparations is essential for the selection of optimal formulations, given their high variability. For instance, dronabinol in a solution exhibits lower intra-individual variability and a faster onset of detectable concentrations compared to capsule formulations [24,50].

We found that most studies on oral PK are focused on synthetic forms and analogues of THC, without considering the other cannabinoids usually present in plant-derived products, thus disregarding their possible therapeutic contributions. Moreover, the presence of CBD and other cannabinoids contained in oral cannabis preparations may be involved in alterations of THC PK properties [14].

In 2017, a review examining the PK and pharmacodynamics (PD) of oral cannabinoids for the treatment of chemotherapy-induced nausea and vomiting emphasized the high variability in the PK/PD profiles of capsules [63] and how they differ from other routes, such as smoking and intravenous delivery. The authors remarked on the efficacy of oral cannabinoids in the management of nausea and vomiting, which is similar, or even superior, to conventional antiemetic drugs. Interestingly, participants showed a preference for cannabis-based medicines over conventional medicines in trials where the two options were compared.

Similarly, a systematic review was recently conducted on CBD PK in humans, regardless of the administration route. Contrary to THC, CBD PK has been more thoroughly studied after oral and oromucosal administration (e.g., oral capsules and oromucosal sprays) than other routes of administration, such as smoking or vaporization. The most commonly studied form of administration was oromucosal spray, which contained CBD in combination with THC. Indeed, the administration of CBD alone in cannabinoid PK studies appeared less frequently than THC alone. Thus, like THC, the authors concluded that the limited available data presented some discrepancies in the PK of CBD [64].

The main limitation of the present systematic review is its use of only the PubMed database, the inclusion of only publications in English, and the exclusion of studies on the oromucosal sprays nabilone and those that administer only CBD.

\section{Conclusions}

In conclusion, oral THC has a highly variable PK profile, which differs between formulations, with seemingly higher variability in baked goods and oil forms. Considering the rapidly changing landscape of medical cannabis laws, there is an evident need for solid PK data after oral administration, especially in dosage forms other than capsules. Particularly, there is a lack of PK data on decoctions (tea) and oils, which are recommended methods of ingestion for medical use. Insufficient studies may lead to future failures of cannabis as a therapeutic compound if its therapeutic window is not defined.

The present review collects all published data on the oral administration of THC and cannabis in humans. Our results show high variability between oral formulations but a positive dose-concentration relationship for THC in most preparations.

Further investigations are required to provide more data on cannabinoid PK in the oral administration of THC, as well as other cannabinoids, to increase the accuracy when defining a therapeutic dosage for every patient.

Author Contributions: Conceptualization, L.P., A.P.P.-A., E.P., C.P.-M., A.S., M.T., F.P.B., and M.F.; methodology, L.P., A.P.P.-A., M.T., and M.F.; data curation, L.P., A.P.P.-A., S.M., O.H., A.S., and M.F.; writing-original draft preparation, L.P., A.P.P.-A., and M.F.; writing-review and editing, all authors. All authors have read and agreed to the published version of the manuscript.

Funding: The investigation was partially carried out with the funding of grants from the Instituto de Salud Carlos III (ISCIII, Fondo Investigación sanitaria (FIS)-Fondo Europeo de Desarrollo Regional (FEDER), FIS PI14/00715 and FIS PI17/01962, predoctoral PFIS FI18/00179 (L. Poyatos), ISCIII-Red de Trastornos Adictivos RTA RD16/0017/0003 and RD16/0017/0010), Ministerio de Sanidad, Política Social e Igualdad (Plan Nacional Sobre Drogas-PNSD, 2015I054 and 2019I010), and Agencia de Gestión de Ayudas Universitarias y de Investigación (AGAUR) Gencat Suport Grups Recerca (2017 SGR 316 and 2017 SGR 530). 
Conflicts of Interest: The authors declare no conflict of interest.

\section{References}

1. United Nations Office on Drugs and Crime (UNODC). World Drug Report 2019. Available online: https://wdr.unodc.org/wdr2019/index.html. (accessed on 5 April 2020).

2. Morales, P.; Hurst, D.P.; Reggio, P.H. Molecular Targets of the Phytocannabinoids: A Complex Picture. Prog. Chem. Org. Nat. Prod. 2017, 103, 103-131. [PubMed]

3. ElSohly, M.A.; Radwan, M.M.; Gul, W.; Chandra, S.; Galal, A. Phytochemistry of Cannabis sativa L. Prog. Chem. Org. Nat. Prod. 2017, 103, 1-36. [PubMed]

4. Kaur, R.; Ambwani, S.R.; Singh, S. Endocannabinoid System: A Multi-Facet Therapeutic Target. Curr. Clin. Pharmacol. 2016, 11, 110-117. [CrossRef]

5. Maccarrone, M.; Maldonado, R.; Casas, M.; Henze, T.; Centonze, D. Cannabinoids therapeutic use: What is our current understanding following the introduction of THC, THC:CBD oromucosal spray and others? Expert Rev. Clin. Pharmacol. 2017, 10, 443-455. [CrossRef]

6. Andre, C.M.; Hausman, J.F.; Guerriero, G. Cannabis sativa: The Plant of the Thousand and One Molecules. Front. Plant. Sci. 2016, 7, 19. [CrossRef] [PubMed]

7. Pisanti, S.; Malfitano, A.M.; Ciaglia, E.; Lamberti, A.; Ranieri, R.; Cuomo, G.; Abate, M.; Faggiana, G.; Proto, M.C.; Fiore, D.; et al. Cannabidiol: State of the art and new challenges for therapeutic applications. Pharmacol. Ther. 2017, 175, 133-150. [CrossRef]

8. Mannucci, C.; Navarra, M.; Calapai, F.; Spagnolo, E.V.; Busardò, F.P.; Cas, R.D.; Ippolito, F.M.; Calapai, G. Neurological Aspects of Medical Use of Cannabidiol. CNS Neurol. Disord. Drug Targets 2017, 16, 541-553. [CrossRef]

9. Laprairie, R.B.; Bagher, A.M.; Kelly, M.E.M.; Denovan-Wright, E.M. Cannabidiol Is a Negative Allosteric Modulator of the Cannabinoid CB1 Receptor. Br. J. Pharmacol. 2015, 172, 4790-4805. [CrossRef]

10. Mueller, J.K.; Reuter, A.R.; Lange, B.; Schaefer, A.; Hanke, F.; Pahlisch, F.; Schaefer, C.; Schmidt, A.-M.; Woelfl, T.; Enning, F.; et al. Effects and interaction of delta-9-tetrahydrocannabidiol and cannabidiol on psychopathology, neurocognition, and endocannabinoids in serum of healthy volunteers: Influence on psychopathology. Neuropsychopharmacology 2016, 41, S589.

11. Morgan, C.J.A.; Freeman, T.P.; Hindocha, C.; Schafer, G.; Gardner, C.; Curran, H.V. Individual and combined effects of acute delta-9tetrahydrocannabinol and cannabidiol on psychotomimetic symptoms and memory function. Transl. Psychiatry 2018, 8, 181. [CrossRef]

12. Hindley, G.; Beck, K.; Borgan, F.; Ginestet, C.E.; McCutcheon, R.; Kleinloog, D.; Ganesh, S.; Radhakrishnan, R.; D'Souza, D.C.; Howes, O.D. Psychiatric symptoms caused by cannabis constituents: A systematic review and meta-analysis. Lancet Psychiatry 2020, 7, 344-353. [CrossRef]

13. Arkell, T.R.; Lintzeris, N.; Kevin, R.C.; Ramaekers, J.G.; Vandrey, R.; Irwin, C.; Haber, P.S.; McGregor, I.S. Cannabidiol (CBD) content in vaporized cannabis does not prevent tetrahydrocannabinol (THC)-induced impairment of driving and cognition. Psychopharmacology 2019, 236, 2713-2724. [CrossRef] [PubMed]

14. Papaseit, E.; Pérez-Mañá, C.; Pérez-Acevedo, A.P.; Hladun, O.; Torres-Moreno, M.C.; Muga, R.; Torrens, M.; Farré, M. Cannabinoids: From pot to lab. Int. J. Med. Sci. 2018, 6, 1286-1295. [CrossRef] [PubMed]

15. Hazekamp, A.; Heerdink, E.R. The prevalence and incidence of medicinal cannabis on prescription in The Netherlands. Eur. J. Clin. Pharmacol. 2013, 69, 1575-1580. [CrossRef]

16. de Hoop, B.; Heerdink, E.R.; Hazekamp, A. Medicinal Cannabis on Prescription in The Netherlands: Statistics for 2003-2016. Cannabis Cannabinoid Res. 2018, 3, 54-55. [CrossRef]

17. Shiplo, S.; Asbridge, M.; Leatherdale, S.T.; Hammond, D. Medical cannabis use in Canada: Vapourization and modes of delivery. Harm Reduct. J. 2016, 13, 30. [CrossRef]

18. Boehnke, K.F.; Scott, J.R.; Litinas, E.; Sisley, S.; Clauw, D.J.; Goesling, J.; Williams, D.A. Cannabis Use Preferences and Decision-making Among a Cross-sectional Cohort of Medical Cannabis Patients with Chronic Pain. J. Pain 2019, 20, 1362-1372. [CrossRef]

19. Bouso, J.C.; Jiménez-Garrido, D.; Ona, G.; Woźnica, D.; Dos Santos, R.G.; Hallak, J.E.C.; Paranhos, B.A.P.B.; de Almeida Mendes, F.; Yonamine, M.; Alcázar-Córcoles, M.Á.; et al. Quality of life, mental health, personality, and patterns of use in self-medicated cannabis users with chronic diseases: A 12-month longitudinal study. Phytother Res. 2020, 1-8. [CrossRef] 
20. Jett, J.; Stone, E.; Warren, G.; Cummings, K.M. Cannabis Use, Lung Cancer, and Related Issues. J. Thorac. Oncol. 2018, 3, 480-487. [CrossRef]

21. Abeles, M.; Popofsky, S.; Wen, A.; Valsamis, C.; Webb, A.; Halaby, C.; Pirzada, M. Vaping-associated lung injury caused by inhalation of cannabis oil. Pediatr. Pulmonol. 2020, 55, 226-228. [CrossRef]

22. Thakrar, P.D.; Boyd, K.P.; Swanson, C.P.; Wideburg, E.; Kumbhar, S.S. E-cigarette, or vaping, product use-associated lung injury in adolescents: A review of imaging features. Pediatr. Radiol. 2020, 50, 338-344. [CrossRef] [PubMed]

23. Duffy, B.; Li, L.; Lu, S.; Durocher, L.; Dittmar, M.; Delaney-Baldwin, E.; Panawennage, D.; LeMaster, D.; Navarette, K.; Spink, D. Analysis of Cannabinoid-Containing Fluids in Illicit Vaping Cartridges Recovered from Pulmonary Injury Patients: Identification of Vitamin E Acetate as a Major Diluent. Toxics 2020, 8, 8. [CrossRef]

24. Brunetti, P.; Pichini, S.; Pacifici, R.; Busardó, F.P.; del Rio, A. Herbal preparations of medical cannabis: A Vademecum for prescribing doctors. Medicina 2020, 56, 237. [CrossRef]

25. Levinsohn, E.A.; Hill, K.P. Clinical uses of cannabis and cannabinoids in the United States. J. Neurol. Sci. 2020, 411, 116717. [CrossRef] [PubMed]

26. Torres-Moreno, M.C.; Papaseit, E.; Torrens, M.; Farré, M. Assessment of Efficacy and Tolerability of Medicinal Cannabinoids in Patients with Multiple Sclerosis: A Systematic Review and Meta-analysis. JAMA Netw. Open 2018, 1, e183485. [CrossRef]

27. EMCDDA (European Monitoring Centre for Drugs and Drug Addiction). Medical Use of Cannabis and Cannabinoids. December 2018. Available online: http://www.emcdda.europa.eu/publications/rapidcommunications/medical-use-of-cannabis-and-cannabinoids-questions-and-answers-for-policymaking en. (accessed on 5 April 2020).

28. Bedrocan Medicinal Cannabis. Available online: https://www.bedrocan.com/ (accessed on 5 April 2020).

29. Ministero della Salute. Decreto 9 novembre 2015: Funzioni di Organismo Statale per la Cannabis previsto dagli articoli 23 e 28 della convenzione unica sugli stupefacenti del 1961, come modificata nel 1972. Available online: https://www.gazzettaufficiale.it/eli/id/2015/11/30/15A08888/sg (accessed on 5 April 2020).

30. Pacifici, R.; Marchei, E.; Salvatore, F.; Guandalini, L.; Busardò, F.P.; Pichini, S. Evaluation of cannabinoids concentration and stability in standardized preparations of cannabis tea and cannabis oil by ultra-high performance liquid chromatography tandem mass spectrometry. Clin. Chem. Lab. Med. 2017, 55, 1555-1563. [CrossRef]

31. Pacifici, R.; Marchei, E.; Salvatore, F.; Guandalini, L.; Busardò, F.P.; Pichini, S. Stability of cannabinoids in cannabis FM1 flowering tops and oil preparation evaluated by ultra-high performance liquid chromatography tandem mass spectrometry. Clin. Chem. Lab. Med. 2019, 57, e165-e168. [CrossRef] [PubMed]

32. Ministero della Salute. La produzione nazionale di sostanze attive di origine vegetale a base di Cannabis. Available online: http://www.salute.gov.it/portale/temi/p2_6.jsp?lingua=italiano\&id=4588\& area=sostanzeStupefacenti\&menu=organismo (accessed on 5 April 2020).

33. Cannimed Medical Cannabis. Available online: https://www.cannimed.ca/ (accessed on 5 April 2020).

34. Romano, L.L.; Hazekamp, A. Cannabis Oil: Chemical evaluation of an upcoming cannabis-based medicine. Cannabinoids 2013, 1, 1-11.

35. Monte, A.A.; Shelton, S.K.; Mills, E.; Saben, J.; Hopkinson, A.; Sonn, B.; Devivo, M.; Chang, T.; Fox, J.; Brevik, C.; et al. Acute Illness Associated with Cannabis Use, by Route of Exposure: An Observational Study. Mol. Med. 2019, 116, 229. [CrossRef]

36. Moher, D.; Liberati, A.; Tetzlaff, J.; Altman, D.G. Preferred reporting items for systematic reviews and meta-analyses: The PRISMA statement. Ann. Int. Med. 2009, 151, 264-269. [CrossRef]

37. Wall, M.E.; Sadler, B.M.; Brine, D.; Taylor, H.; Perez-Reyes, M. Metabolism, disposition, and kinetics of delta-9-tetrahydrocannabinol in men and women. Clin. Pharmacol. Ther. 1983, 34, 352-363. [CrossRef] [PubMed]

38. Haney, M.; Bisaga, A.; Foltin, R.W. Interaction between naltrexone and oral THC in heavy marijuana smokers. Psychopharmacology 2003, 166, 77-85. [CrossRef] [PubMed]

39. Naef, M.; Curatolo, M.; Petersen-Felix, S.; Arendt-Nielsen, L.; Zbinden, A.; Brenneisen, R. The analgesic effect of oral delta-9-tetrahydrocannabinol (THC), morphine, and a THC-morphine combination in healthy subjects under experimental pain conditions. Pain 2003, 105, 79-88. [CrossRef] 
40. Guy, G.W.; Robson, P.J. A Phase I, Open Label, Four-Way Crossover Study to Compare the Pharmacokinetic Profiles of a Single Dose of $20 \mathrm{mg}$ of a Cannabis Based Medicine Extract (CBME) Administered on 3 Different Areas of the Buccal Mucosa and to Investigate the Pharmacokinetics of CBME per Oral in Healthy Male and Female Volunteers (GWPK0112). J. Cannabis Ther. 2004, 3, 79-120.

41. Ménétrey, A.; Augsburger, M.; Favrat, B.; Pin, M.A.; Rothuizen, L.E.; Appenzeller, M.; Buclin, T.; Mangin, P.; Giroud, C. Assessment of driving capability through the use of clinical and psychomotor tests in relation to blood cannabinoids levels following oral administration of $20 \mathrm{mg}$ dronabinol or of a cannabis decoction made with 20 or $60 \mathrm{mg}$ Delta9-THC. J. Anal. Toxicol. 2005, 29, 327-338. [CrossRef] [PubMed]

42. Nadulski, T.; Sporkert, F.; Schnelle, M.; Stadelmann, A.M.; Roser, P.; Schefter, T.; Pragst, F. Simultaneous and sensitive analysis of THC, 11-OH-THC, THC-COOH, CBD, and CBN by GC-MS in plasma after oral application of small doses of THC and cannabis extract. J. Anal. Toxicol. 2005, 29, 782-789. [CrossRef]

43. Goodwin, R.S.; Gustafson, R.A.; Barnes, A.; Nebro, W.; Moolchan, E.T.; Huestis, M.A. Delta(9)-tetrahydrocannabinol, 11-hydroxy-delta(9)-tetrahydrocannabinol and 11-nor-9-carboxy-delta(9)-tetrahydrocannabinol in human plasma after controlled oral administration of cannabinoids. Ther. Drug Monit. 2006, 28, 545-551. [CrossRef]

44. Schwilke, E.W.; Schwope, D.M.; Karschner, E.L.; Lowe, R.H.; Darwin, W.D.; Kelly, D.L.; Goodwin, R.S.; Gorelick, D.A.; Huestis, M.A. Delta9-tetrahydrocannabinol (THC), 11-hydroxy-THC, and 11-nor-9-carboxy-THC plasma pharmacokinetics during and after continuous high-dose oral THC. Clin. Chem. 2009, 55, 2180-2189. [CrossRef]

45. Karschner, E.L.; Darwin, W.D.; Goodwin, R.S.; Wright, S.; Huestis, M.A. Plasma cannabinoid pharmacokinetics following controlled oral delta9-tetrahydrocannabinol and oromucosal cannabis extract administration. Clin. Chem. 2011, 57, 66-75. [CrossRef]

46. Karschner, E.L.; Schwope, D.M.; Schwilke, E.W.; Goodwin, R.S.; Kelly, D.L.; Gorelick, D.A.; Huestis, M.A. Predictive model accuracy in estimating last $\Delta$ 9-tetrahydrocannabinol (THC) intake from plasma and whole blood cannabinoid concentrations in chronic, daily cannabis smokers administered subchronic oral THC. Drug Alcohol Depend. 2012, 125, 313-319. [CrossRef]

47. Martin-Santos, R.; Crippa, J.A.; Batalla, A.; Bhattacharyya, S.; Atakan, Z.; Borgwardt, S.; Allen, P.; Seal, M.; Langohr, K.; Farre, M.; et al. Acute effects of a single, oral dose of d9-tetrahydrocannabinol (THC) and cannabidiol (CBD) administration in healthy volunteers. Curr. Pharm. Des. 2012, 18, 4966-4979. [CrossRef]

48. Eichler, M.; Spinedi, L.; Unfer-Grauwiler, S.; Bodmer, M.; Surber, C.; Luedi, M.; Drewe, J. Heat exposure of Cannabis sativa extracts affects the pharmacokinetic and metabolic profile in healthy male subjects. Planta Med. 2012, 78, 686-691. [CrossRef]

49. Lile, J.A.; Kelly, T.H.; Charnigo, R.J.; Stinchcomb, A.L.; Hays, L.R. Pharmacokinetic and pharmacodynamic profile of supratherapeutic oral doses of $\Delta(9)$-THC in cannabis users. J. Clin. Pharmacol. 2013, 53, 680-690. [CrossRef] [PubMed]

50. Parikh, N.; Kramer, W.G.; Khurana, V.; Cognata Smith, C.; Vetticaden, S. Bioavailability study of dronabinol oral solution versus dronabinol capsules in healthy volunteers. Clin. Pharmacol. 2016, 12, 155-162. [CrossRef] [PubMed]

51. Cherniakov, I.; Izgelov, D.; Barasch, D.; Davidson, E.; Domb, A.J.; Hoffman, A. Piperine-pro-nanolipospheres as a novel oral delivery system of cannabinoids: Pharmacokinetic evaluation in healthy volunteers in comparison to buccal spray administration. J. Control. Release 2017, 28, 1-7. [CrossRef] [PubMed]

52. Pellesi, L.; Licata, M.; Verri, P.; Vandelli, D.; Palazzoli, F.; Marchesi, F.; Cainazzo, M.M.; Pini, L.A.; Guerzoni, S. Pharmacokinetics and tolerability of oral cannabis preparations in patients with medication overuse headache (MOH)-a pilot study. Eur J. Clin. Pharmacol. 2018, 74, 1427-1436. [CrossRef]

53. Pichini, S.; Mannocchi, G.; Gottardi, M.; Pérez-Acevedo, A.P.; Poyatos, L.; Papaseit, E.; Pérez-Mañá, C.; Farré, M.; Pacifici, R.; Busardò, F.P. Fast and sensitive UHPLC-MS/MS analysis of cannabinoids and their acid precursors in pharmaceutical preparations of medical cannabis and their metabolites in conventional and non-conventional biological matrices of treated individual. Talanta 2020, 209, 120537. [CrossRef]

54. Timpone, J.G.; Wright, D.J.; Li, N.; Egorin, M.J.; Enama, M.E.; Mayers, J.; Galetto, G. The safety and pharmacokinetics of single-agent and combination therapy with megestrol acetate and dronabinol for the treatment of HIV wasting syndrome. The DATRI 004 Study Group. Division of AIDS Treatment Research Initiative. AIDS Res. Hum. Retroviruses 1997, 13, 305-315. [CrossRef] [PubMed] 
55. Klumpers, L.E.; Beumer, T.L.; van Hasselt, J.G.; Lipplaa, A.; Karger, L.B.; Kleinloog, H.D.; Freijer, J.I.; de Kam, M.L.; van Gerven, J.M. Novel $\Delta$-(9)-tetrahydrocannabinol formulation Namisol ${ }^{\circledR}$ has beneficial pharmacokinetics and promising pharmacodynamic effects. Br. J. Clin. Pharmacol. 2012, 74, 42-53. [CrossRef]

56. Ahmed, A.I.; van den Elsen, G.A.; Colbers, A.; van der Marck, M.A.; Burger, D.M.; Feuth, T.B.; Rikkert, M.G.; Kramers, C. Safety and pharmacokinetics of oral delta-9-tetrahydrocannabinol in healthy older subjects: A randomized controlled trial. Eur. Neuropsychopharmacol. 2014, 24, 1475-1482. [CrossRef]

57. de Vries, M.; Van Rijckevorsel, D.C.; Vissers, K.C.; Wilder-Smith, O.H.; Van Goor, H. Single dose delta-9-tetrahydrocannabinol in chronic pancreatitis patients: Analgesic efficacy, pharmacokinetics and tolerability. Br. J. Clin. Pharmacol. 2016, 81, 525-537. [CrossRef] [PubMed]

58. Ohlsson, A.; Lindgren, J.E.; Wahlen, A.; Agurell, S.; Hollister, L.E.; Gillespie, H.K. Plasma delta-9 tetrahydrocannabinol concentrations and clinical effects after oral and intravenous administration and smoking. Clin. Pharmacol. Ther. 1980, 28, 409-416. [CrossRef] [PubMed]

59. Wachtel, S.R.; ElSohly, M.A.; Ross, S.A.; Ambre, J.; de Wit, H. Comparison of the subjective effects of Delta(9)-tetrahydrocannabinol and marijuana in humans. Psychopharmacology 2002, 161, 331-339. [PubMed]

60. Newmeyer, M.N.; Swortwood, M.J.; Barnes, A.J.; Abulseoud, O.A.; Scheidweiler, K.B.; Huestis, M.A. Free and Glucuronide Whole Blood Cannabinoids' Pharmacokinetics after Controlled Smoked, Vaporized, and Oral Cannabis Administration in Frequent and Occasional Cannabis Users: Identification of Recent Cannabis Intake. Clin. Chem. 2016, 62, 1579-1592. [CrossRef]

61. Newmeyer, M.N.; Swortwood, M.J.; Andersson, M.; Abulseoud, O.A.; Scheidweiler, K.B.; Huestis, M.A. Cannabis Edibles: Blood and Oral Fluid Cannabinoid Pharmacokinetics and Evaluation of Oral Fluid Screening Devices for Predicting $\Delta$ 9-Tetrahydrocannabinol in Blood and Oral Fluid following Cannabis Brownie Administration. Clin. Chem. 2017, 63, 647-662. [CrossRef]

62. Vandrey, R.; Herrmann, E.S.; Mitchell, J.M.; Bigelow, G.E.; Flegel, R.; LoDico, C.; Cone, E.J. Pharmacokinetic Profile of Oral Cannabis in Humans: Blood and Oral Fluid Disposition and Relation to Pharmacodynamic Outcomes. J. Anal. Toxicol. 2017, 41, 83-99. [CrossRef]

63. Badowski, M.E. A review of oral cannabinoids and medical marijuana for the treatment of chemotherapy-induced nausea and vomiting: A focus on pharmacokinetic variability and pharmacodynamics. Cancer Chemother. Pharmacol. 2017, 80, 441-449. [CrossRef]

64. Millar, S.A.; Stone, N.L.; Yates, A.S.; O'Sullivan, S.E. A Systematic Review on the Pharmacokinetics of Cannabidiol in Humans. Front. Pharmacol. 2018, 9, 1365. [CrossRef] 